\title{
Defect Detection in Textured Materials Using Optimized Filters
}

\author{
Ajay Kumar, Member, IEEE, and Grantham K. H. Pang, Senior Member, IEEE
}

\begin{abstract}
The problem of automated defect detection in textured materials is investigated. A new approach for defect detection using linear FIR filters with optimized energy separation is proposed. Performance of different feature separation criterion with reference to fabric defects has been evaluated. The issues relating to the design of optimal filters for supervised and unsupervised web inspection are addressed. A general web inspection system based on the optimal filters is proposed. The experiments on this new approach have yielded excellent results. The low computational requirement confirms the usefulness of the approach for industrial inspection.
\end{abstract}

Index Terms-Defect detection, industrial inspection, optimized FIR filters, performance evaluation, quality assurance, textured defects.

\section{INTRODUCTION}

A UTOMATED visual inspection of industrial materials such as textile, paper, and plastic requires adaptive solutions that can be executed in real time. Currently, the quality assurance of web processing is mainly carried out by manual inspection. However, manual inspection is labor intensive and insufficient to maintain high quality standards at high-speed production. For example, in the textile industry, only about $70 \%$ of defects are being detected by manual inspection even with the highly trained inspectors [1]. Therefore, automation of visual inspection tasks can increase the efficiency of production lines and improve quality of product as well.

Industrial web inspection has extremely high requirements and is most challenging as compared to other inspection problems [2]. A typical web is $1.5-2 \mathrm{~m}$ wide and is processed at the speed of 8-20 m per minute. Consequently, the throughput for $100 \%$ inspection is tremendous and therefore most feasible solutions require additional hardware components and reduction in calculation complexity. At microscopic level, broad spectrum of different web inspection problems reduce to texture analysis problems.

In last 20 years, defect detection in textured materials has been studied using several approaches (Table I). The detailed description of these approaches is beyond the scope of this paper (due to space limitations) and therefore only selective references are provided. However, the detailed description of these approaches can be found in [3].

Manuscript received April 29, 2000; revised June 24, 2001. This paper was recommended by Associate Editor V. Murino.

A. Kumar is with the Department of Computer Science, Hong Kong University of Science and Technology, Clear Water Bay, Hong Kong (e-mail: ajaykr@cs.ust.hk).

G. K. H. Pang is with the Department of Electrical and Electronic Engineering, The University of Hong Kong, Hong Kong.

Publisher Item Identifier S 1083-4419(02)06046-6.
TABLE I

Methods For the Detection of Defects in TeXtured Materials

\begin{tabular}{|c|c|c|}
\hline Approach & Method & $\overline{\text { References }}$ \\
\hline \multirow{10}{*}{ Statistical } & 1. Fractal dimension & [4] \\
\hline & 2. Bi-level thresholding & {$[5-[7]$} \\
\hline & 3. Gray-level statistics & {$[8]-[10]$} \\
\hline & 4. Morphological operations & {$[8],[11]$} \\
\hline & 5. Edge detection & {$[12]-[13]$} \\
\hline & 6. Normalized cross-correlation & [14]-[15] \\
\hline & 7. Co-occurrence matrix & {$[16]-[19]$} \\
\hline & 8. Eigenfilters & [20] \\
\hline & 9. Local linear transforms & {$[21]-[22]$} \\
\hline & 10. Rank-order functions & {$[23]-[24]$} \\
\hline \multirow{8}{*}{ Spectral } & 1. Discrete Fourier transform & {$[25]-[27]$} \\
\hline & 2. Optical Fourier transform & {$[28]-[30]$} \\
\hline & 3. Windowed Fourier transform & {$[31]-[32]$} \\
\hline & 4. Gabor filter & {$[33]-[34]$} \\
\hline & 5. Real Gabor function & {$[35]$} \\
\hline & 6. Wavelet transform & {$[1]-[2],[36]-[37]$} \\
\hline & 7. Wavelet packets & {$[38]$} \\
\hline & 8. Wigner-distribution & [55] \\
\hline \multirow{3}{*}{ Model-based } & 1. Gauss Markov random field & {$[39]-[40]$} \\
\hline & 2. Poissonian model & {$[41]-[42]$} \\
\hline & 3. Model-based clustering & [43] \\
\hline
\end{tabular}

\section{A. Proposed Approach}

The Gabor filters and the infinite impulse response (IIR) filters are the filters with only a few free parameters and therefore the search space for optimization is very restricted. Better optimization results can be obtained when the number of free available parameters of a filter is large. A general finite impulse response filter (FIR) has generally more free parameters than an IIR or a Gabor filter. The single biggest advantage of FIR filters is that they can implement any impulse response, provided it is of finite length. Despite its several advantages, the design of FIR filters for the defect detection has not been attempted, as can be seen from the literature review presented in [3] or in Table I.

In this paper, a new approach to the fabric defect detection using linear FIR filters with optimized energy separation is investigated. The fabric textures are modeled by their autocorrelation functions. The linear FIR filters that guarantee optimal discrimination of energy in local regions rather than optimal representation are used in this work. These optimal filters cannot 
explicitly detect the defects but can make the detection an easier task by greatly attenuating pixel value in the defect-free region relative to regions having defects. The closed-form solution suggested by Mahalanobis-Singh [44], [45] and related approach by Unser [46] has been investigated for defect detection. The approximate closed-form solution suggested by Randen and Husøy [47], [48] for optimal energy separation using Fisher criterion [49] will also be used. The main contributions from this paper [3] are summarized as follows.

1) A new approach for the defect detection in textured materials using linear FIR filters with the optimized energy separation is proposed.

2) The performance of three feature separation criterions proposed by Mahalanobis-Singh [44], [45], Unser [46] and Fisher [49] has been evaluated on real fabric samples. Two quantitative measures, i.e., minimum size mask and the misclassification rate, have been introduced in Section III-E for the performance evaluation of the optimal filters designed with different object functions.

3) The performance of the proposed defect detection approach with the variation in the size of 1) optimal filter and 2) smoothing filter is investigated.

4) One of the important conclusions of this work is that the size of optimal filter has appreciable effect on the performance for the defect detection. Prior work [47], [48] on texture segmentation has not accounted for the size of optimal filter mask and therefore some of the conclusions in prior research have been subjective to the size of optimal filter masks used (more details in Section III-E and Section V).

5) Another approach for unsupervised defect detection for inspection of web materials using optimal filters is presented. Excellent fabric defect detection results, obtained using a simple two-optimal-filter model, are demonstrated.

Prior texture segmentation work [47], [48] using optimal filters has been concentrated on Brodatz album. However, the present work on defect segmentation pertains to real meaningful patterns from the textile industry. In this paper, the term "optimal filter" refers to the linear FIR filter with the optimized energy separation.

\section{MATHEMATICAL FOUNDATIONS}

The feature extraction model used to design optimal filters is illustrated in Fig. 1. This model was proposed by Jain et al. [50] and has been used in several references [44], [45], [47], [48]. The objective of the optimal filter $h_{o p}(x, y)$ is to extract those frequencies where the defect-free texture has low signal energy and the texture with defect has high signal energy. If this is accomplished, the defective regions in the composite inspection image can be segmented by the analysis of the local texture energy.

\section{A. Local Energy Estimate}

The energy of pixels in a gray-level image $w(x, y)$ is defined as $E\left\{w^{2}(x, y)\right\}$, where $E$ stands for the expectation. The defects in the inspection images are (assumed to be) local rather than global. Therefore, a local operator is needed to compute the energy of an image $w(x, y)$ in the local region, i.e., the local energy estimate. This is accomplished by smoothing the image $w^{2}(x, y)$ as shown in Fig. 1. Unser [51] has suggested that the squaring $|\cdot|^{2}$ operation in conjunction with $\log |\cdot|$ (second nonlinearity) after the smoothing gives the best operator pair, from a set of several other tested operators, for the unsupervised texture segmentation. The classifier used in this work is a thresholding operator. Therefore the application of second monotonic transformation $\log |\cdot|$, to the extracted features, will not have any effect in the classification (thresholding) of features. Therefore the second nonlinearity has not been employed in this work.

In this model, it is assumed that the fabric textures being modeled are wide sense stationary and that they can be well described by their autocorrelation functions. Let $x$ and $y$ be the spatial indices of acquired image $I(x, y)$. As shown in Fig. 1, filtering of acquired image $I(x, y)$ with filter $h_{o p}(x, y)$ generates a new image $w(x, y)$ :

$$
\begin{aligned}
w(x, y) & =h_{o p}(x, y)^{*} I(x, y) \\
& =\sum_{m=0}^{M-1} \sum_{n=0}^{N-1} h_{o p}(m, n) I(x-m, y-n)
\end{aligned}
$$

where $\left(^{*}\right)$ denotes the 2-D convolution and $h_{o p}(x, y)$ is an $M \times$ $N$ optimal filter to be designed. For every pixel in $w(x, y)$, the output can be rewritten as

$$
w(x, y)=\mathbf{h}_{o p}^{T} \mathbf{i}(x, y)
$$

where $\mathbf{h}_{o p}$ and $\mathbf{i}(x, y)$ are the vectors of length $Z=M \times N$, obtained by lexicographical ordering of columns of $h_{o p}(x, y)$ and $M \times N$ window of $I(x, y)$ around pixel $(x, y)$ respectively:

$$
\begin{aligned}
& \mathbf{i}(x, y)=\left[\begin{array}{c}
I(x, y) \\
\cdot . \\
\ddot{y} \\
I(x, y-N+1) \\
I(x-1, y) \\
. \cdot \\
\ddot{1} \\
I(x-M+N-N+1)
\end{array}\right] \\
& \mathbf{h}_{o p}=\left[\begin{array}{c}
h_{o p}(0,0) \\
\ddot{.} \\
h_{o p}(0, N-1) \\
h_{o p}(1,0) \\
\ddot{.} \\
h_{o p}(M-1, N-1)
\end{array}\right] .
\end{aligned}
$$

The squaring nonlinear operator $|\cdot|^{2}$ computes the energy of every pixel in the filtered image $w(x, y)$ :

$$
s(x, y)=w^{2}(x, y) .
$$

The energy of pixels in the image $s(x, y)$ is now calculated with reference to a local region (determined by the bandwidth of smoothing filter) around pixels at $(x, y)$. The local energy estimate $f(x, y)$ is obtained by smoothing the image $s(x, y)$ with a filter $g(x, y)$, i.e.,

$$
\begin{aligned}
f(x, y) & =g(x, y)^{*} s(x, y) \\
& =\mathbf{g}^{T} \mathbf{s}(x, y) .
\end{aligned}
$$




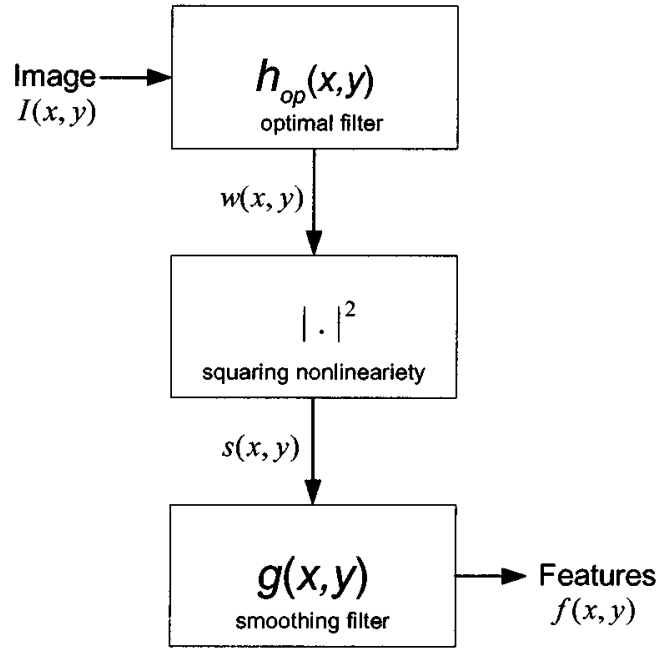

Fig. 1. Block diagram of the feature extraction model.

The filtered image $f(x, y)$ is referred to as the feature image in the following discussion.

1) The Feature Mean: The inspection images are assumed to be random process, which are wide sense stationary (WSS) over the (bounded) region of interest. Using the vector formulation for the filtering operation, the mean value of the feature image $f(x, y)$ can be derived as follows [47], [48]:

$$
\begin{aligned}
\mu_{f} & =E\{f(x, y)\} \\
& =E\left\{s(x, y)^{*} g(x, y)\right\} .
\end{aligned}
$$

The smoothing filter $g(x, y)$ is a unity gain low pass filter. Therefore, the mean feature value at the output of this filter is equal to mean feature value at the input. Assuming that the filter coefficients are such that $\sum_{x, y} g(x, y)=1,(6)$ can be written as

$$
\begin{aligned}
\mu_{f} & =E\{s(x, y)\}=E\left\{w^{2}(x, y)\right\} \\
& =E\left\{\left(\mathbf{h}_{o p}^{T} \mathbf{i}(x, y)\right)\left(\mathbf{h}_{o p}^{T} \mathbf{i}(x, y)\right)\right\} \\
& =\mathbf{h}_{o p}^{T} E\left\{\mathbf{i}(x, y) \mathbf{i}^{T}(x, y)\right\} \mathbf{h}_{o p}
\end{aligned}
$$

defining

$$
\mathbf{R}_{i i}=E\left\{\mathbf{i}(x, y) \mathbf{i}^{T}(x, y)\right\} .
$$

Equation (7) can be written as

$$
\mu_{f}=\mathbf{h}_{o p}^{T} \mathbf{R}_{i i} \mathbf{h}_{o p}
$$

where $\mathbf{R}_{i i}$ defined in the (8) is the autocorrelation matrix of the image $\mathbf{i}(x, y)$. The autocorrelation matrix of the vector $\mathbf{i}(x, y)$ can be easily constructed from the two-dimensional (2-D) autocorrelation function of the image $\mathbf{i}(x, y)$ [47]. Since the $R_{i i}$ in (8) is symmetric, the derivative of mean feature value $\mu_{f}$ is given by

$$
\frac{\partial \mu_{f}}{\partial \mathbf{h}_{o p}}=\frac{\partial\left(\mathbf{h}_{o p}^{T} \mathbf{R}_{i i} \mathbf{h}_{o p}\right)}{\partial \mathbf{h}_{o p}}=2 \mathbf{R}_{i i} \mathbf{h}_{o p} .
$$

Equations (9) and (10) will be used, in the designing of optimal filters using the closed-form optimization, in the subsequent sections.
2) The Feature Variance: The variance of the feature image $f(x, y)$ is given by

$$
\begin{aligned}
\sigma_{f}^{2} & =E\left\{\left(f(x, y)-\mu_{f}\right)^{2}\right\} \\
& =E\left\{f^{2}(x, y)\right\}-2 \mu_{f} E\{f(x, y)\}+\mu_{f}^{2} \\
& =E\left\{f^{2}(x, y)\right\}-\mu_{f}^{2} .
\end{aligned}
$$

Using (5), the above equation can be written as

$$
\begin{aligned}
\sigma_{f}^{2} & =E\left\{\left(\mathbf{g}^{T} \mathbf{s}(x, y)\right)\left(\mathbf{g}^{T} \mathbf{s}(x, y)\right)\right\}-\mu_{f}^{2} \\
& =\mathbf{g}^{T} \mathbf{R}_{s s} \mathbf{g}-\mu_{f}^{2}
\end{aligned}
$$

where

$$
\mathbf{R}_{s s}=E\left\{\mathbf{s}(x, y) \mathbf{s}^{T}(x, y)\right\} .
$$

The $Z \times Z$ autocorrelation matrix $\mathbf{R}_{s s}$ can be readily constructed from the autocorrelation function of the image $s(x, y)$. By approximating the inspection images as separable autoregressive process [52] of order one, the simplified expressions for the variance and its derivatives can be developed. These expressions have been derived in reference [47] and can be written as follows:

$$
\begin{gathered}
\sigma_{f}^{2} \approx 2 \lambda_{f}\left(\mathbf{h}_{o p}^{T} \mathbf{R}_{i i} \mathbf{h}_{o p}\right)^{2} \\
\frac{\partial \sigma_{f}^{2}}{\partial \mathbf{h}_{o p}} \approx 8 \lambda_{f}\left(\mathbf{h}_{o p}^{T} \mathbf{R}_{i i} \mathbf{h}_{o p}\right) \mathbf{R}_{i i} \mathbf{h}_{o p}
\end{gathered}
$$

where $\lambda_{f}$ is some scalar, such that $\partial \lambda_{f} / \partial \mathbf{h}_{o p} \approx 0$.

\section{B. Object Functions for Optimization}

The objective of designing optimal filters is to locate the defects in the textured images under inspection. The response of such an optimal filter to the inspection image with defects should be strong, i.e., high $\mu_{f_{d}}$. On the other hand, when a defect-free inspection image, is presented to this optimal filter, its response should be low i.e., low $\mu_{f_{r}}$. Thus the obvious aim of the optimal filter is to achieve the maximum separation between the average local energies, i.e., $\mu_{f_{d}}$ and $\mu_{f_{r}}$, at the filter output.

Mahalanobis and Singh [44], [45] have proposed that the ratio between the expected energies (average feature values) of the filter response can be used as a quality measure for the separation of features. The criterion function

$$
J_{1}\left(\mathbf{h}_{o p}\right)=\frac{\mu_{f_{d}}}{\mu_{f_{r}}}
$$

was used in [44] and [45] for the optimization of a linear FIR filter, i.e., selection of an optimal filter. The measure of relative distance between the average feature values can also be used for the optimization of a single filter with respect to the discrimination between two textures. The object function

$$
J_{2}\left(\mathbf{h}_{o p}\right)=\frac{\left(\mu_{f_{d}}-\mu_{f_{r}}\right)^{2}}{\mu_{f_{d}} \mu_{f_{r}}}
$$

was originally suggested by Unser [46] for the design of optimal texture transforms. The design technique suggested by Unser [46] cannot be directly used for the filter design, since the texture transform corresponds to the sub-sampled filter-bank. 
However, Randen and Husøy [47], [53] have adapted this object function for the designing of optimal filters for the texture segmentation.

The main disadvantage of the object functions $J_{1}\left(\mathbf{h}_{o p}\right)$ and $J_{2}\left(\mathbf{h}_{o p}\right)$ is that the variances of the features, $\sigma_{f_{d}}$ and $\sigma_{f_{r}}$, are not taken into account. Consequently, the optimal filters designed with respect to these object functions can only achieve large separation of feature means $\mu_{f_{d}}$ and $\mu_{f_{r}}$. But if the variance of local energy estimate $\sigma_{f_{d}}$ and $\sigma_{f_{r}}$ are large, then the feature distribution can considerably overlap. Therefore an optimal filter should not only produce large separation of mean local energy estimate, but also yield low variances $\sigma_{f_{d}}$ and $\sigma_{f_{r}}$. An object function that is commonly used in the pattern recognition literature is the Fisher criterion [49], which also takes variances of the feature distributions into account.

$$
J_{3}\left(\mathbf{h}_{o p}\right)=\frac{\left(\mu_{f_{d}}-\mu_{f_{r}}\right)^{2}}{\sigma_{f_{d}}^{2}+\sigma_{f_{r}}^{2}} .
$$

The mathematical framework for designing the optimal filters with respect to these three criterion functions will be reviewed in the following three sections.

1) Optimization Using $J_{1}\left(\mathbf{h}_{o p}\right)$ as the Object Function: From (9) it can be noted that the average feature value, $\mu_{f_{d}}$ and $\mu_{f_{r}}$, is a function of the filter coefficient vector $\mathbf{h}_{o p}$. Therefore, (15) can be written as

$$
J_{1}\left(\mathbf{h}_{o p}\right)=\frac{\mu_{f_{d}}}{\mu_{f_{r}}}=\frac{\mathbf{h}_{o p}^{T} \mathbf{R}_{i i_{d}} \mathbf{h}_{o p}}{\mathbf{h}_{o p}^{T} \mathbf{R}_{i i_{r}} \mathbf{h}_{o p}}
$$

finding the filter coefficient vector, $\mathbf{h}_{o p}$, corresponding to the maximum object function $J_{1}\left(\mathbf{h}_{o p}\right)$, entails the solution of

$$
\begin{aligned}
\frac{\partial J_{1}\left(\mathbf{h}_{o p}\right)}{\partial \mathbf{h}_{o p}} & =0, \text { or } \\
\frac{2 \mathbf{R}_{i i_{d}} \mathbf{h}_{o p}}{\mathbf{h}_{o p}^{T} \mathbf{R}_{i i_{r}} \mathbf{h}_{o p}}-\frac{2 \mathbf{h}_{o p}^{T} \mathbf{R}_{i i_{d}} \mathbf{h}_{o p} \mathbf{R}_{i i_{r}} \mathbf{h}_{o p}}{\left[\mathbf{h}_{o p}^{T} \mathbf{R}_{i i_{r}} \mathbf{h}_{o p}\right]^{2}} & =0 .
\end{aligned}
$$

Let

$$
\psi=\frac{\mathbf{h}_{o p}^{T} \mathbf{R}_{i i_{d}} \mathbf{h}_{o p}}{\mathbf{h}_{o p}^{T} \mathbf{R}_{i i_{r}} \mathbf{h}_{o p}} .
$$

Equation (19) yields

$$
\mathbf{R}_{i i_{r}}^{-1} \mathbf{R}_{i i_{d}} \mathbf{h}_{o p}=\psi \cdot h_{o p} .
$$

This is an eigenvalue equation where the filter $\mathbf{h}_{o p}$ is the eigenvector and $\psi$ is the eigenvalue. The expression for eigenvalue $\psi$ is identical to $J_{1}\left(\mathbf{h}_{o p}\right)$, i.e., $\psi=J_{1}\left(\mathbf{h}_{o p}\right)$, the object function to be optimized. Therefore, the optimal filter is the eigenvector $\mathbf{h}_{o p}$ that yields maximum object function $J_{1}\left(\mathbf{h}_{o p}\right)$. The choice of the weakest eigenvector corresponding to smallest eigenvalue (object function) generates inverse solution, i.e., gray levels in filtered image are interchanged from maximum to minimum [44].

2) Optimization Using $J_{2}\left(\mathbf{h}_{o p}\right)$ as the Object Function: The filter optimization with respect to the object function $J_{2}\left(\mathbf{h}_{o p}\right)$ maximizes the squared difference of average feature values, normalized by the product of average feature values. The optimization with respect to $J_{2}\left(\mathbf{h}_{o p}\right)$ entails the solution of

$$
\frac{\partial J_{2}\left(\mathbf{h}_{o p}\right)}{\partial \mathbf{h}_{o p}}=0
$$

using (23) and the chain rule of the differentation, the above equation yields

$$
\frac{\partial J_{2}\left(\mathbf{h}_{o p}\right)}{\partial \mu_{f_{d}}} \frac{\partial \mu_{f_{d}}}{\partial \mathbf{h}_{o p}}+\frac{\partial J_{2}\left(\mathbf{h}_{o p}\right)}{\partial \mu_{f_{r}}} \frac{\partial \mu_{f_{r}}}{\partial \mathbf{h}_{o p}}=0 .
$$

Substituting from (9) and (10) in (22), we get

$$
\mathbf{R}_{i i_{r}}^{-1} \mathbf{R}_{i i_{d}} \mathbf{h}_{o p}=\psi \cdot \mathbf{h}_{o p}
$$

where again

$$
\psi=\frac{\mu_{f_{d}}}{\mu_{f_{r}}} .
$$

The (23) is the same eigenvalue problem as (21) in the previous optimization approach. Thus in this approach also, the eigenvector yielding the maximum object function is selected as the coefficients of the required optimal filter $\mathbf{h}_{o p}$.

Since $J_{2}\left(\mathbf{h}_{o p}\right)=\psi+\psi^{-1}-2$, the eigenvalues in (23)-(24) are used for the selection of corresponding eigenvector. In general $J_{1}\left(\mathbf{h}_{o p}\right) \neq J_{2}\left(\mathbf{h}_{o p}\right)$, therefore the eigenvector selected by this approach, as the optimal filter $\mathbf{h}_{o p}$, can be different from those computed by the previous approach in Section II-B1.

3) Optimization Using $J_{3}\left(\mathbf{h}_{o p}\right)$ as the Object Function: As in the previous two cases, the optimal filter that maximizes the object function $J_{3}\left(\mathbf{h}_{o p}\right)$ can be found by equating the partial derivative of $J_{3}\left(\mathbf{h}_{o p}\right)$ to zero, i.e.

$$
\begin{gathered}
\frac{\partial J_{3}\left(\mathbf{h}_{o p}\right)}{\partial \mathbf{h}_{o p}}=0, \text { or } \\
2\left(\sigma_{f_{d}}^{2}+\sigma_{f_{r}}^{2}\right) \frac{\partial \mu_{f_{d}}}{\partial \mathbf{h}_{o p}}-\left(\mu_{f_{d}}-\mu_{f_{r}}\right) \frac{\partial \sigma_{f_{d}}^{2}}{\partial \mathbf{h}_{o p}} \\
=2\left(\sigma_{f_{d}}^{2}+\sigma_{f_{r}}^{2}\right) \frac{\partial \mu_{f_{r}}}{\partial \mathbf{h}_{o p}}+\left(\mu_{f_{d}}-\mu_{f_{r}}\right) \frac{\partial \sigma_{f_{r}}^{2}}{\partial \mathbf{h}_{o p}} .
\end{gathered}
$$

Randen and Husøy [47] have developed a closed-form solution for the above equation using the approximate expressions of variance and its derivative. By substituting the expression for mean and its derivative from (9) and (10) and variance expressions from (13) and (14), (25) can be simplified as

$$
\begin{aligned}
\left(\lambda_{f_{r}}\left(\mathbf{h}_{o p}^{T} \mathbf{R}_{i i_{r}} \mathbf{h}_{o p}\right)^{2}\right. & +\lambda_{f_{d}}\left(\mathbf{h}_{o p}^{T} \mathbf{R}_{i i_{d}} \mathbf{h}_{o p}\right) \\
& \left.\cdot\left(\mathbf{h}_{o p}^{T} \mathbf{R}_{i i_{r}} \mathbf{h}_{o p}\right)\right) \mathbf{R}_{i i_{d}} \mathbf{h}_{o p} \\
= & \left(\lambda_{f_{d}}\left(\mathbf{h}_{o p}^{T} \mathbf{R}_{i i_{d}} \mathbf{h}_{o p}\right)^{2}\right. \\
& +\lambda_{f_{r}}\left(\mathbf{h}_{o p}^{T} \mathbf{R}_{i i_{d}} \mathbf{h}_{o p}\right) \\
& \left.\cdot\left(\mathbf{h}_{o p}^{T} \mathbf{R}_{i i_{r}} \mathbf{h}_{o p}\right)\right) \mathbf{R}_{i i_{r}} \mathbf{h}_{o p}
\end{aligned}
$$

which can be further simplified as

$$
\begin{aligned}
\left(\mathbf{R}_{i i_{r}}^{-1} \mathbf{R}_{i i_{d}}\right) \mathbf{h}_{o p} & =\frac{\left(\mathbf{h}_{o p}^{T} \mathbf{R}_{i i_{d}} \mathbf{h}_{o p}\right)}{\left(\mathbf{h}_{o p}^{T} \mathbf{R}_{i i_{r}} \mathbf{h}_{o p}\right)} \mathbf{h}_{o p}, \text { or } \\
\mathbf{R}_{i i_{r}}^{-1} \mathbf{R}_{i i_{d}} \mathbf{h}_{o p} & =\psi \cdot \mathbf{h}_{o p} .
\end{aligned}
$$

Equation (26) is identical to the eigenvalue problem discussed in the previous approaches, i.e., (21) and (23). Thus the coefficients of the optimal filter can be obtained from the eigenvectors of $\left(\mathbf{R}_{i i_{r}}^{-1} \mathbf{R}_{i i_{d}}\right)$ which generates the maximum object function $J_{3}\left(\mathbf{h}_{o p}\right)$. 
To sum up, the optimal filters corresponding to three object functions suggested by Mahalanobis-Singh [44], [45], Unser [46] and Fisher [49] are computed as follows.

1) The correlation matrices $\mathbf{R}_{\ddot{i}_{r}}$ and $\mathbf{R}_{i i_{d}}$ from fabric samples are computed.

2) The eigenvectors of $\left(\mathbf{R}_{i i_{r}}^{-1} \mathbf{R}_{i i_{d}}\right)$ are computed.

3) The eigenvector yielding maximum object function $\left(J_{1}\left(\mathbf{h}_{o p}\right), J_{2}\left(\mathbf{h}_{o p}\right)\right.$, or $\left.J_{3}\left(\mathbf{h}_{o p}\right)\right)$ is selected.

4) Optimal filter $h_{o p}(x, y)$ is obtained from elements of $\mathbf{h}_{o p}$, by inverse lexicographical reordering.

\section{SuPERVISED DEFECT DETECTION}

In most industrial inspection problems, the priori knowledge of defect to be detected is available. Inspection of such known defects can be regarded as supervised defect detection [3], [34]. For some known category of defects in textile webs, specific optimal filters can be designed to detect those defects. The selection of parameters for designing these optimal filters and their performance on the defect detection has been investigated in the following sections.

\section{A. Size of the Optimal Filter}

The dimensions of an FIR filter are related to its bandwidth. Filters for large bandwidth require smaller dimension and viceversa [44]. Appropriate dimensions of optimal filter can be determined from the spectral characteristics of fabric image, which is related to yarn density and weaving pattern. For a plain weave fabric, if the yarn density is high, then its image pixels will become uncorrelated rapidly. This means that the spectrum of such fabric has probably high frequency content that require large bandwidth or spatial filter of lower dimension. Similarly, filters of large spatial dimension are needed for the fabrics with lower yarn density. However, the spectral characteristics of a fabric defect can be entirely different from its defect-free background, i.e., yarn density. Therefore, above all, as will be seen in Section III-E, the size of an optimal filter depends on the spectral characteristics of the defect to be detected. A symmetric region of support is required for accurate edge localization and therefore, only odd sized filter masks are designed.

\section{B. Selection of a Smoothing Filter}

An important element in optimal filter design is the choice of smoothing filter. The objective of a smoothing filter is to transform areas of high local band pass energy to strong gray level distributions. Among several candidate filters, Gaussian low pass smoothing filter is commonly used [47], [48], [50], [51] since it is separable and offers optimal joint resolution in spatial frequency and spatial domain:

$$
g(x, y)=\frac{1}{\sqrt{2 \pi} \gamma} e^{-\left((1 / 2)\left(x^{2}+y^{2}\right) / \gamma^{2}\right)}
$$

The choice of bandwidth $\gamma$ determines the frequencies to be included for local energy estimation at the output. Finite approximation of the above filter (in (27) implemented as separable convolution masks are used as smoothing filter. As proposed by Jain et al. [50] the choice

$$
\gamma=\frac{1}{2 \sqrt{2} f_{0}}
$$

is most appropriate and is used for all experiments shown in this paper. The choice of center frequency $f_{0}$ depends on the number of pixels occupied by one yarn in an image. For all twill fabric samples shown in this paper, one yarn occupies approximately eight pixels and therefore $f_{0}=1 / 8$ is chosen. While filtering the image near the image boundary, the image is assumed to be extended by its mirror image, i.e., with even reflections.

\section{Experimental Setup}

Samples of the most commonly occurring fabric defects in the twill and the plain weave fabrics were gathered from a loom. The images of all these fabric samples were acquired under backlighting condition and covered $1.28 \times 1.28 \mathrm{in}^{2}$ area of the fabric sample. The acquired images were digitized into $256 \times 256$ pixels, with eight-bit resolution. While evaluating the performance for the defect detection, those images were preferred in which the defects were hidden in the background texture and therefore the defect detection was expected to be difficult. In all the experiments, the analyzed images have zero mean and were histogram equalized. A Gaussian low pass filter, as described in Section III-B, was used for smoothing. For all the experimental results reported in this paper, the spatial extent of the Gaussian low pass filter is empirically fixed as $K \times L=11 \times 11$, unless otherwise explicitly stated.

The fabric defects in each of the gathered images are localized in a small region, i.e., they are not global. If the complete $256 \times 256$ pixels image is utilized for designing the optimal filter, the discriminating effect of defect from its large defect-free background diminishes due to the inherent averaging that takes place while computing its correlation matrix $\mathbf{R}_{i i_{d}}$. Furthermore, the computational time for computing the correlation matrices, $\mathbf{R}_{i i_{d}}$ and $\mathbf{R}_{i i_{r}}$, for the complete image is significantly high. Therefore, only a small image pitch from the region of image having defect (and equal sized image pitch from a defect-free image) is utilized for designing optimal filters. The size of this image pitch is empirically determined and it depends on the spatial extent of the defect in an image.

\section{Results}

Fig. 2(a) shows the sample of a twill weave fabric with the defect commonly referred to as mispick. Using $J_{1}\left(\mathbf{h}_{o p}\right)$ as the object function, a $7 \times 7$ optimal filter was designed to segment the defect mispick. Fig. 2(h) shows the possible eigenvalues of (21). The eigenvector corresponding to the largest eigenvalue (4.419) was picked up as the optimal filter. Fig. 2(g) shows the magnitude frequency response of this optimal filter. It can be seen that the magnitude frequency response exhibits passbands where local energy estimate is high (corresponding to defect) and stopbands elsewhere. The filtered image is shown in Fig. 2(b). As seen from this image, the standard deviation of individual pixels corresponding to defect is much higher than those due to defect-free region and, therefore, defect can be segmented by any two class linear discriminant function, typically 


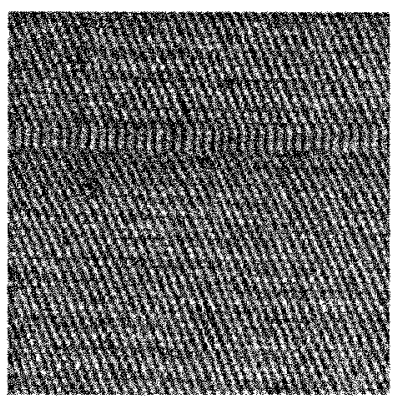

(a)

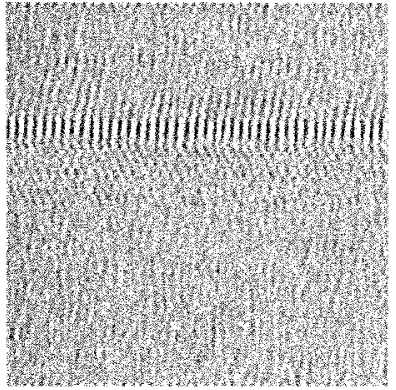

(b)

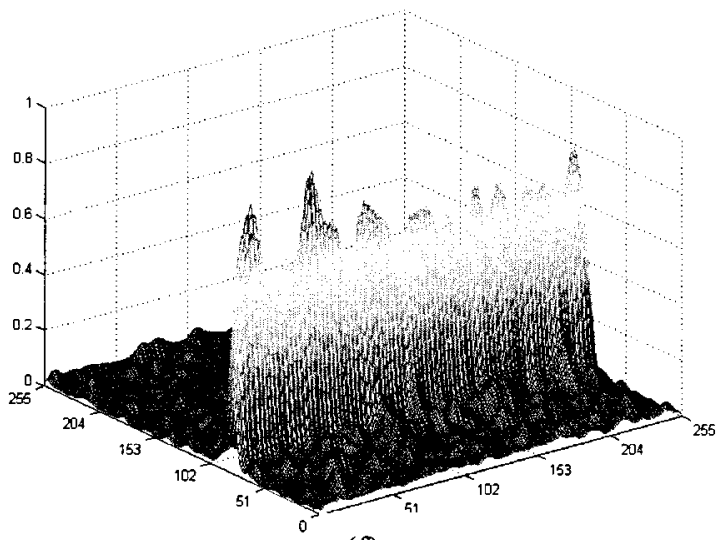

(f)

(c)
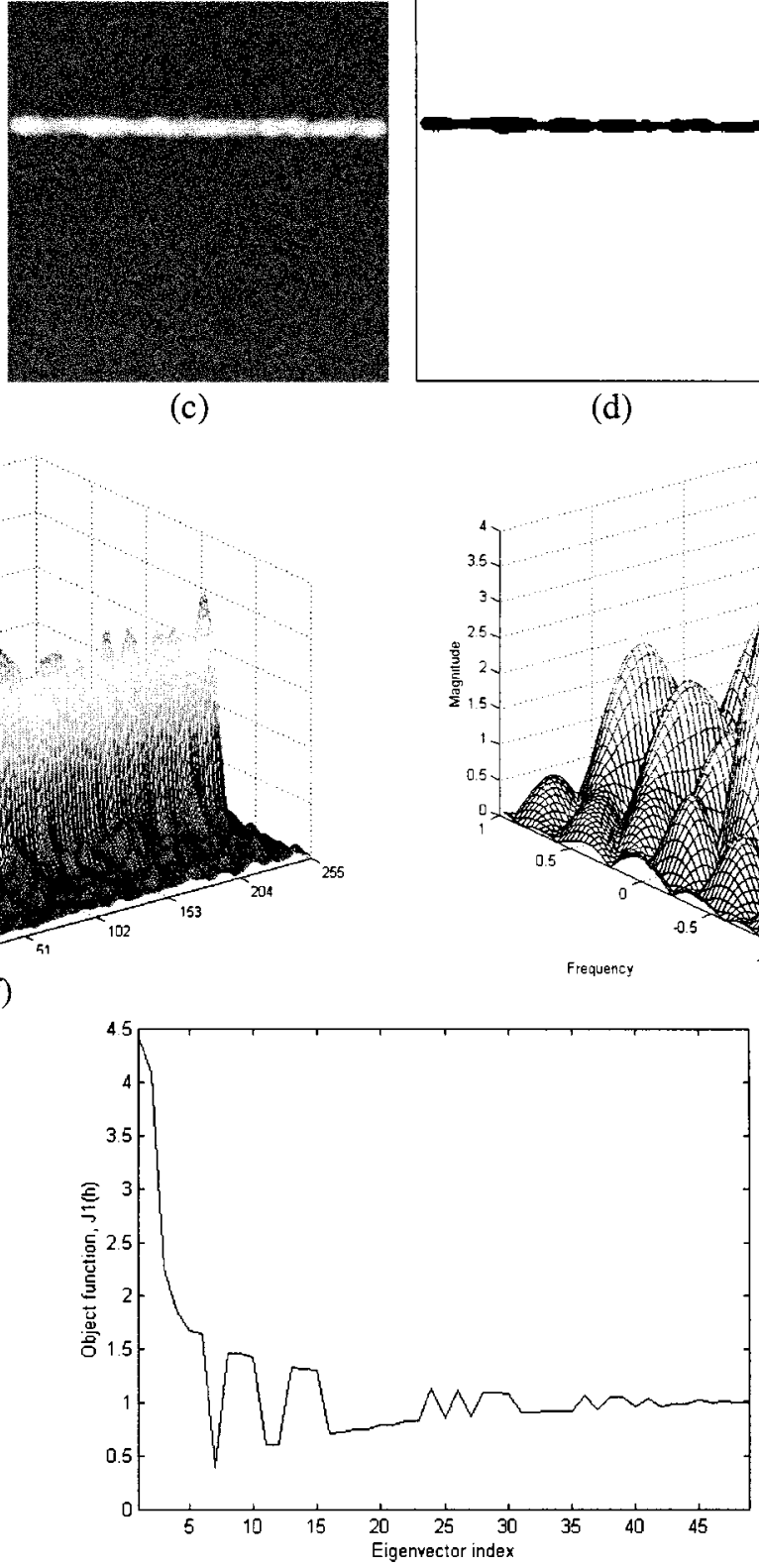

(h)

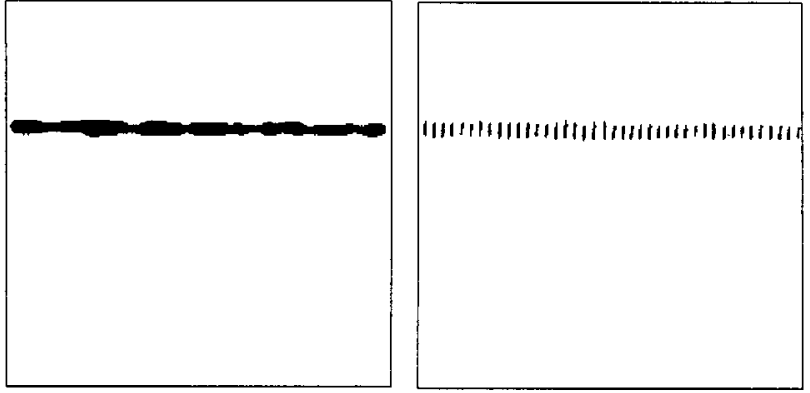

(d)

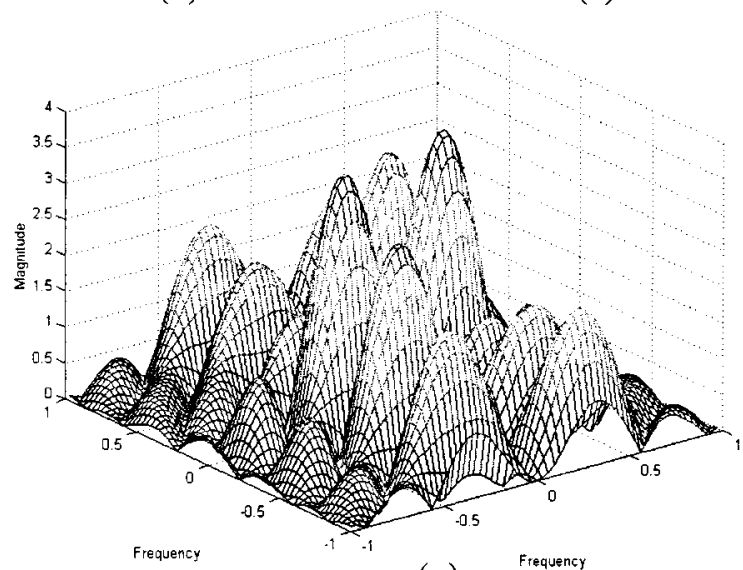

(g)

Fig. 2. (a) Fabric sample with mispick, (b) after filtering with $7 \times 7$ optimal filter, (c) local energy estimate of image in (b), (d) segmented defect after thresholding image (c), (e) segmented defect after thresholding (b), (f) 2-D mesh plot of local energy estimate, (g) amplitude frequency response of $7 \times 7$ optimal filter, and (h) object function for each of the 49 eigenvectors.

thresholding. The segmented defect in the thresholded image is shown in Fig. 2(e). The local energy estimate for the image in Fig. 2(b) is shown in Fig. 2(f). Since the average local en- ergy for region corresponding to defect is 4.419 times (greater than one) that of defect-free region, the defect can be easily be segmented by simple thresholding [Fig. 2(d)]. The optimal fil- 
TABLE II

Maximum Object Function as a Function of Mask Size for the MISPICK SHOWN IN FIG. 2(a)

\begin{tabular}{c|c|c|c}
\hline $\begin{array}{c}\text { Optimal } \\
\text { filter Mask } \\
\text { size }\end{array}$ & $J_{1}\left(\mathbf{h}_{o p}\right)$ & $J_{2}\left(\mathbf{h}_{o p}\right)$ & $J_{3}\left(\mathbf{h}_{o p}\right)$ \\
\hline $3 \times 3$ & 1.4082 & 0.1183 & 0.4063 \\
\hline $5 \times 5$ & 2.8315 & 1.1846 & 1.0283 \\
\hline $7 \times 7$ & 4.4187 & 2.6450 & 0.9406 \\
\hline $9 \times 9$ & 5.5855 & 3.7645 & 1.0117 \\
\hline $11 \times 11$ & 6.6410 & 4.7916 & 1.0602 \\
\hline $13 \times 13$ & 7.8826 & 6.0094 & 0.9269 \\
\hline $15 \times 15$ & 8.8267 & 6.9399 & 1.1859 \\
\hline $17 \times 17$ & 9.8922 & 7.9932 & 1.6401 \\
\hline $19 \times 19$ & 10.8588 & 8.9509 & 1.3436 \\
\hline
\end{tabular}

ters designed in this experiments were found to be robust (as will be shown in Section III-E) and have successfully detected defects of similar nature lying anywhere in the image under inspection. The magnitude of the three object functions, i.e., $J_{1}\left(\mathbf{h}_{o p}\right), J_{2}\left(\mathbf{h}_{o p}\right), J_{3}\left(\mathbf{h}_{o p}\right)$, for the different optimal filter size $(M \times N)$, for the defect in Fig. 2(a) is shown in Table II. With the increase in mask size from $3 \times 3$ to $19 \times 19$, the maximum eigenvalue of (21) or the object function $J_{1}\left(\mathbf{h}_{o p}\right)$ increases linearly. The high magnitude of $J_{1}\left(\mathbf{h}_{o p}\right)$ results in higher attenuation of the defect-free region relative to the region having defect, but is computationally expansive. For the image shown in Fig. 2(a), the optimal filters (eigenvectors) selected by using the object function $J_{1}\left(\mathbf{h}_{o p}\right)$ were same as those for $J_{2}\left(\mathbf{h}_{o p}\right)$, for all the different mask sizes shown in Table II. Therefore, as discussed in Section II-B2, the object function $J_{2}\left(\mathbf{h}_{o p}\right)$ in Table II can be computed directly from $J_{1}\left(\mathbf{h}_{o p}\right)$, e.g., for $7 \times 7$ mask $J_{2}\left(\mathbf{h}_{o p}\right)=4.4187+(4.4187)^{-1}-2=2.6450$.

Fig. 3 shows some fabric samples with different defects and their detection using three different object functions. A plain weave fabric sample with a defect, commonly referred to as wrong-draw, is shown in Fig. 3(a). Three $5 \times 5$ optimal filters, corresponding to three different object functions, were designed to detect these fabric defects. The detection results for these defects are shown in Fig. 3(b)-(d). Fig. 3(e) and 3(m) shows twill weave fabric sample with defect colored-yarn and dirty-yarn respectively. The results for these defects with the corresponding $7 \times 7$ optimal filters are shown in Fig. 3(f)-(h) and 3(n)-(p). The results for the fabric defect colored-yarn in Fig. 3(e) shows that the $7 \times 7$ optimal filter selected by using the object function $J_{3}\left(\mathbf{h}_{o p}\right)$ emphasizes on the defect-free background, while the optimal filter selected by using the other two object functions emphasize on defect and gives better results. Another twill weave fabric sample in which the defect broken-end is visible with great difficulty appears in Fig. 3(i). As shown in Fig. 3(j)-(k) $7 \times 7$ optimal filters designed with object functions $J_{1}\left(\mathbf{h}_{o p}\right)$ and $J_{2}\left(h_{o p}\right)$ fails to detect any defect. However, the use of the Fisher criterion $\left(J_{3}\left(\mathbf{h}_{o p}\right)\right)$ has succeeded, as can be seen in Fig. 3(1). The optimal filters designed for the four images in Fig. 3 were same for the object function $J_{1}\left(\mathbf{h}_{o p}\right)$ and $J_{2}\left(\mathbf{h}_{o p}\right)$. However, this may not be the case always and the different optimal filters can be obtained with these two object functions as will be shown in Section III-F.
The images shown in Fig. 4 depict four different kinds of fabric defects. The detection results for these images, using the optimal filters designed with $J_{2}\left(\mathbf{h}_{o p}\right)$ are shown in Fig. 4. The size of optimal filters used for these images is kept small and is mentioned in the figure captions. The small sized optimal filters, in the range of $3 \times 3$ to $9 \times 9$, have been used with the marginal compromise in the performance (Section III-E). The thresholding limit for these images is computed from a defect-free (reference) fabric image sample [3]. The maximum value of local energy estimate from the reference images, similar to as in (30), is used as thresholding limit.

\section{E. Performance}

The lack of an appropriate criterion for defect detection makes it very difficult to compare the performance of a defect detection technique on various defects. However, a commonly used criterion for the quantification of image segmentation results is the percentage of misclassified pixels. In this work, the misclassification rate $(M R)$ is defined as: the total number of misclassified pixels in the defect-free region (noise), which are expressed as the percentage of the total detected pixels in the defect region. This measure is directly related to the false alarm in the inspection process. However, a zero misclassification rate $(M R)$ does not necessarily mean good defect detection unless it is accompanied by a large number of pixels in the region of image corresponding to the defect. Therefore, whenever required, the total number of pixels $\left(P_{d}\right)$ in the region of image having defect and the total number of pixels $\left(P_{n}\right)$ in the defect-free region can be used for the comparison.

The size of the optimal filter mask required to detect a defect is an important parameter. The smaller value of this mask size $(M \times N)$ permits higher computational savings during the online inspection and therefore should be kept as small as possible. However experimental results in Table II have suggested that in most cases, the optimal filters designed with the object functions $J_{1}\left(\mathbf{h}_{o p}\right)$ and $J_{2}\left(\mathbf{h}_{o p}\right)$, the performance improves when the size of optimal filter is increased (in the range $7 \times 7$ to $19 \times 19$ ). Therefore it is necessary to compute the minimum size of optimal filters that are required to achieve a reasonable level of performance. Table III shows the minimum size of the optimal filter mask required to achieve the misclassification rate $(M R)$ of less than $1 \%$ while detecting the corresponding defects. While obtaining the minimum optimal filter size required in Table III, the size of the Gaussian smoothing filter was kept constant, i.e., $11 \times 11$. It was also noticed that (and will be shown in Table IV) the size of the smoothing filter does not have any effect on the required minimum size of the optimal filter. If $M R<1 \%$ is achieved by the optimal filters designed by using more than one object functions, than the object function achieving higher (if) value of $P_{d}$ is mentioned in Table III.

The results in Table III suggests that the optimal filter mask of size $3 \times 3$ is sufficient to detect the mispick shown in Fig. 2(a). With the increase in mask size from $3 \times 3$ to $5 \times 5(7 \times 7)$ computational time for the filtering increases by $77(340) \%$ but the object function $J_{1}\left(\mathbf{h}_{o p}\right)$, or the relative attenuation of defect-free background, only increases by 1 


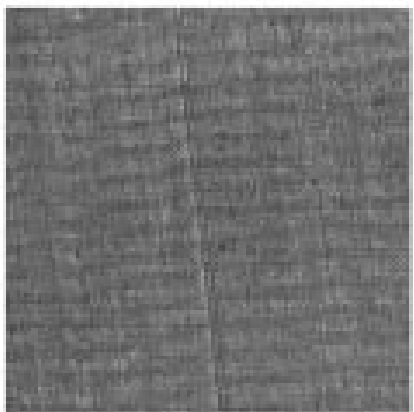

(a)

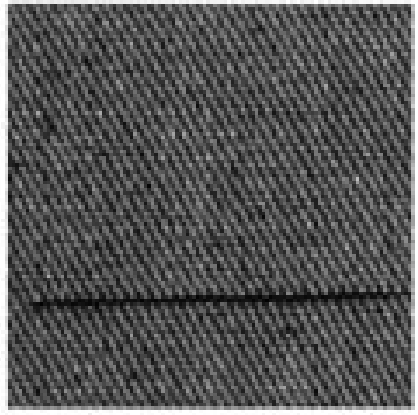

(e)

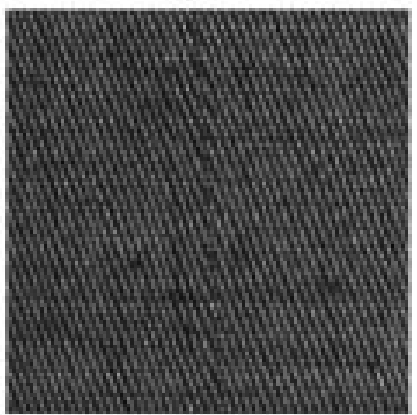

(i)

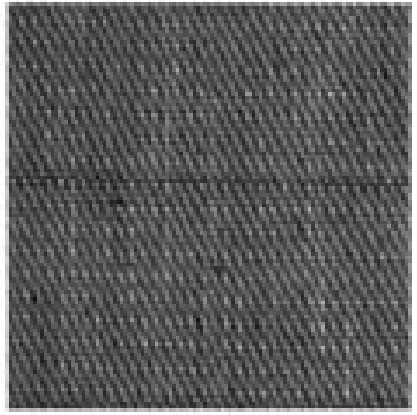

(m)

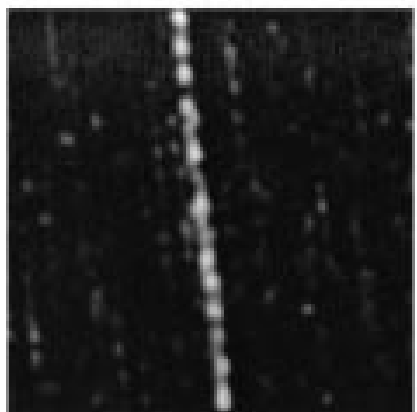

(b)

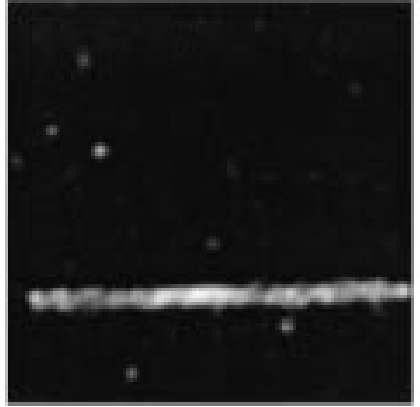

(f)

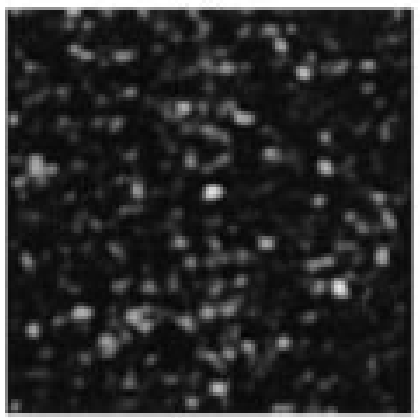

(j)

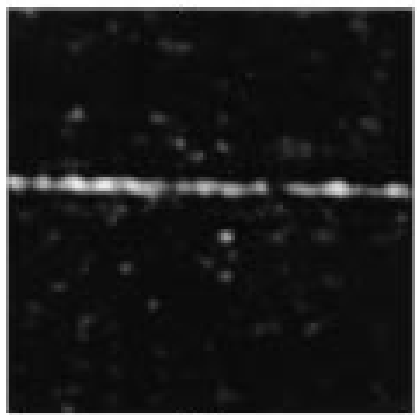

(n)

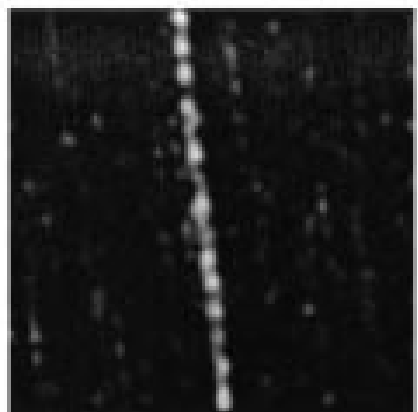

(c)

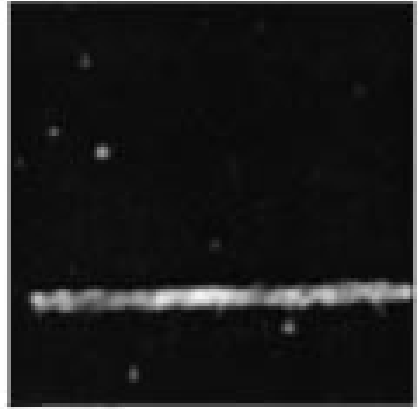

(g)

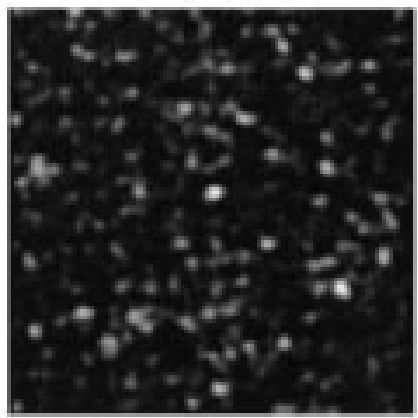

(k)

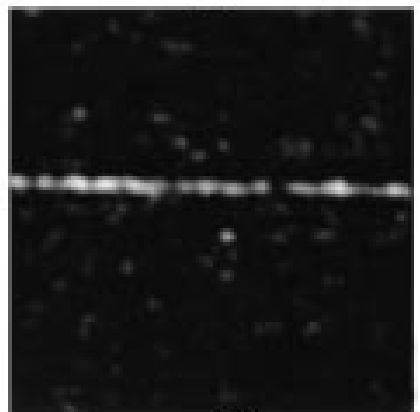

(o)

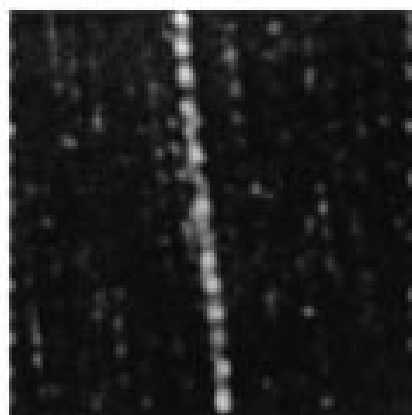

(d)

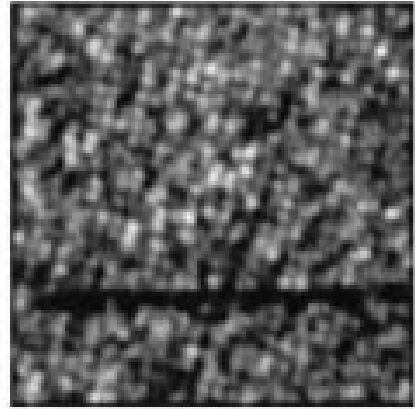

(h)

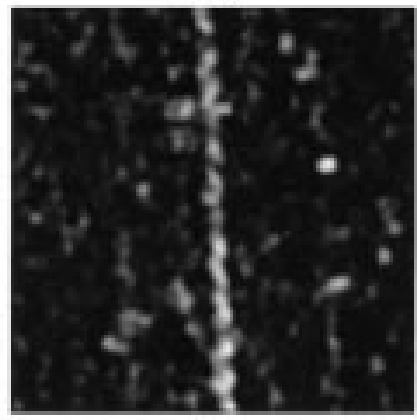

(I)

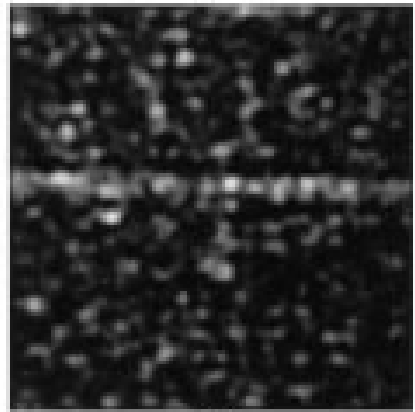

(p)

Fig. 3. Fabric samples with wrong-draw, colored-yarn, broken-end and dirty-yarn in (a), (e), (i), and (m) respectively; corresponding local energy estimates with the optimal filters designed using object function $J_{1}\left(\mathbf{h}_{o} p\right)$ in (b), (f), (j), and (n); object function $J_{2}\left(\mathbf{h}_{o} p\right)$ in $(\mathrm{c})$, (g), (k) and $(\mathrm{o})$; object function $J_{3}\left(\mathbf{h}_{o} p\right)$ function (d), (h), (l), and (p).

(114)\%. The detection of mispick with the $3 \times 3$ mask is shown in Fig. 5. The usage of $7 \times 7$ mask shown in Fig. 2 offers higher attenuation of defect-free background as compared to those for $3 \times 3$ mask and therefore permits less chance of generating false alarm. Fig. 5 shows the robustness of the $3 \times 3$ mask used to obtain the results in Fig. 5(b)-(c), for the detection of other mispick defects in the same direction.

Table III shows the high yarn density plain weave fabric samples require only $5 \times 5$ optimal filter masks. The defect detec- tion using the required $5 \times 5$ masks has already been shown in Figs. 3(a) and 4(b). The twill weave fabric sample also required $5 \times 5$ optimal filter for the detection of big-knot with the $M R$ of $0 \%$. However, the detection of this defect using the $3 \times 3$ optimal filter mask with the $M R$ of $1.24 \%$ has been shown in Fig. 4(b)-(d). The detection results for the defect wrong-draw and dirty-yarn with the minimum required mask size of $7 \times 7$ has been shown in Fig. 3(f)-(g) and Fig. 3(n)-(o), but without the thresholding operation. 


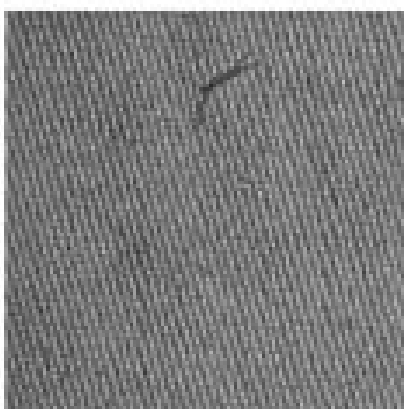

(a)

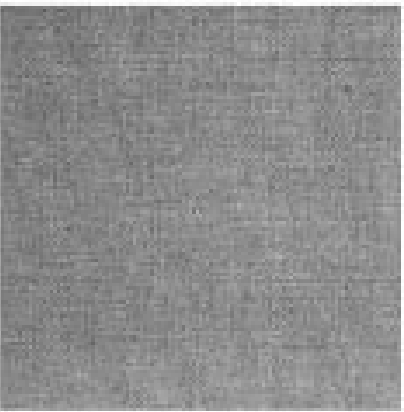

(e)

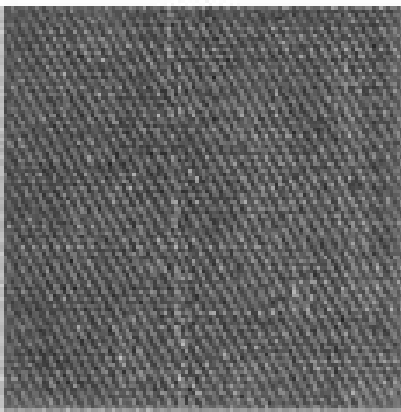

(i)

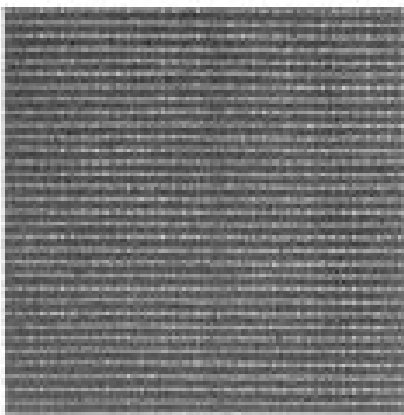

(m)

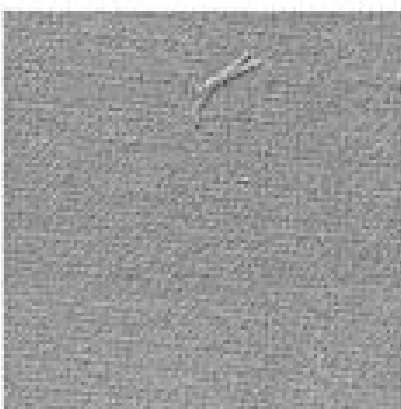

(b)

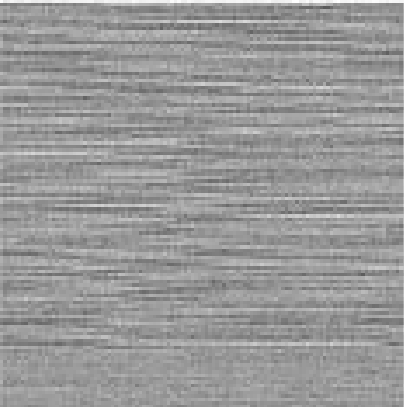

(f)

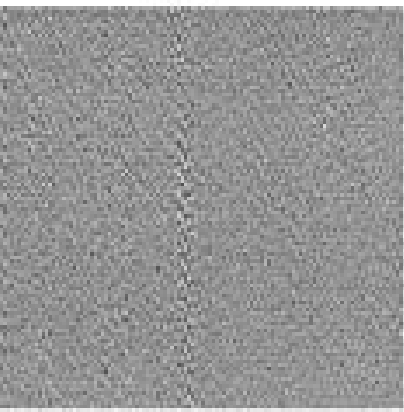

(j)

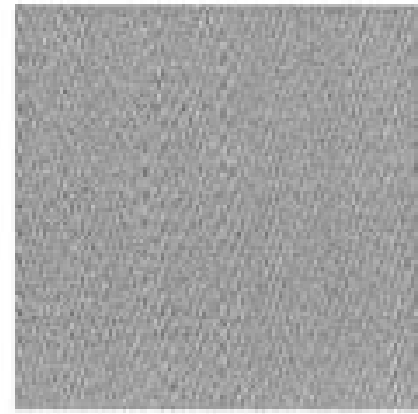

(n)

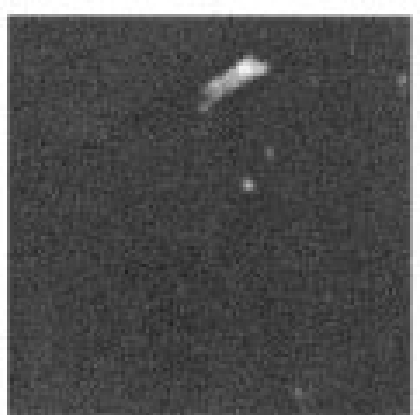

(c)

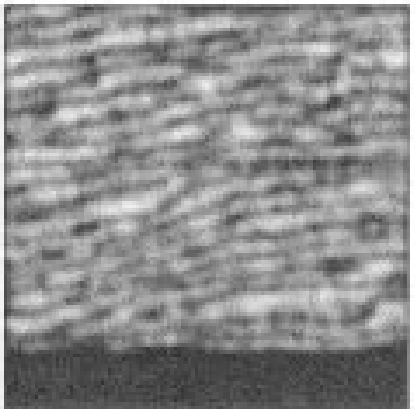

(g)

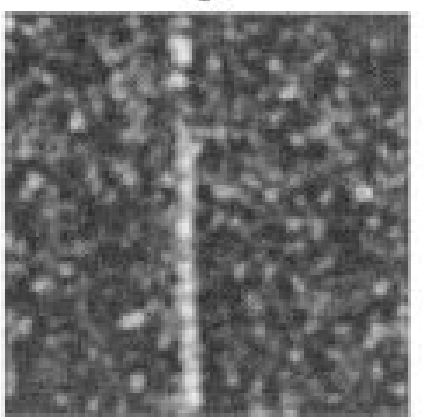

(k)

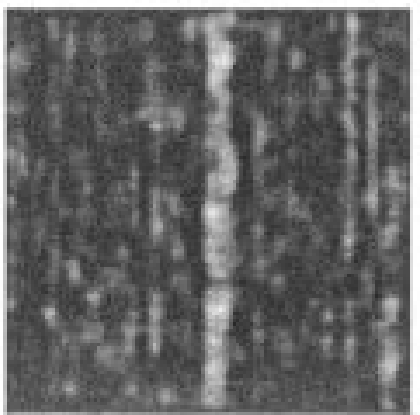

(o)

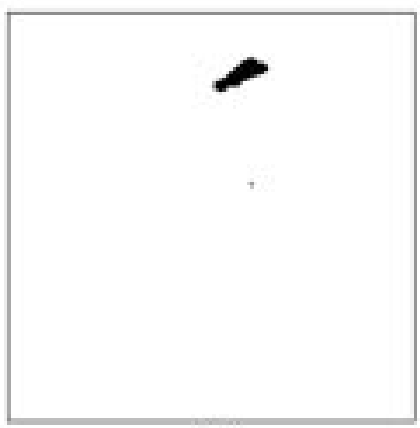

(d)

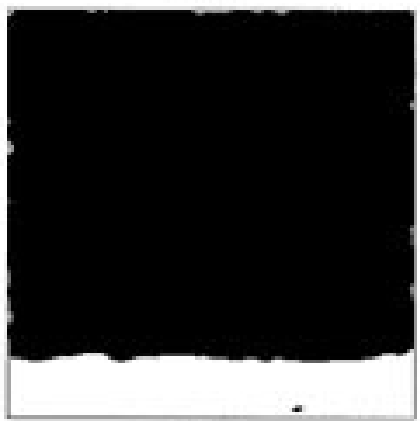

(h)

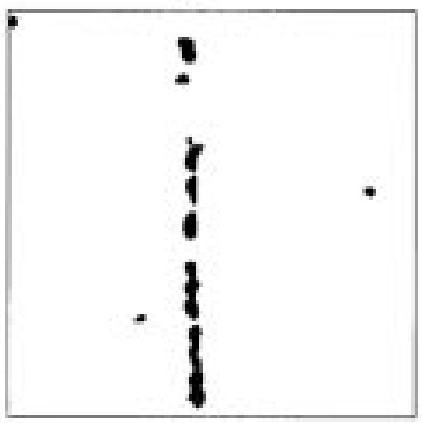

(1)

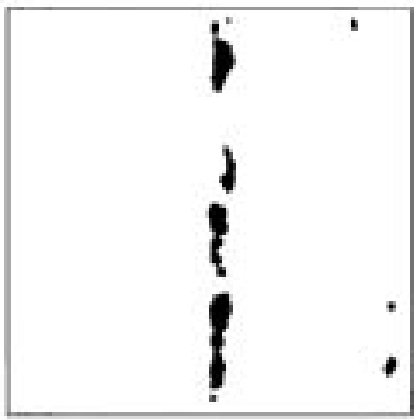

(p)

Fig. 4. Fabric samples with big-knot, double-weft, broken-yarn, and tripe-warp in (a), (e), (i), and (m) respectively; corresponding filtered image with optimal filter of size (b) $3 \times 3$, (f) $5 \times 5$, (j) $7 \times 7$, and (n) $9 \times 9$; the corresponding local energy estimates in (c), (g), (k), and (o); corresponding segmented defects with the misclassification rate (as defined in Section III-E) of (d) $1.24 \%$, (h) $0.03 \%$, (l) $8.26 \%$, and (p) $7.62 \%$.

The detection of fabric defect broken-end, shown in Fig. 3(i), requires the minimum mask size of $11 \times 11$. Thus the detection of this defect with the $7 \times 7$ optimal filter mask, shown in Fig. 3(i)-(f) with $M R$ of $8.26 \%$, can be made much better (noiseless) if the optimal filter mask suggested in Table III is used. Similarly, the noise seen in the thresholded image of Fig. 4(1) can be suppressed when an optimal filter of size $9 \times 9$ is used.
The optimal filters designed with all the three different object function for the image in Fig. 4(i) generated $M R$ of $0 \%$. However, the number of pixels in the defect region, i.e. $P_{d}$, was higher (almost double) for the optimal filters designed with the Fisher criterion, i.e. $J_{3}\left(\mathbf{h}_{o p}\right)$. Therefore only the object function $J_{3}\left(\mathbf{h}_{o p}\right)$ is suggested in Table III. Another example of fabric defect with very subtle intensity variations was shown in Fig. 4(m). 
TABLE III

Minimum Mask Size ReQuired for the Defect Detection With $M R<1 \%$

\begin{tabular}{c|c|c|c|c|c|c|c|c|c}
\hline Mask Size & $\mathbf{3} \times \mathbf{3}$ & \multicolumn{3}{|c|}{$\mathbf{5 \times 5}$} & \multicolumn{2}{|c|}{$7 \times \mathbf{7}$} & $\mathbf{9} \times \mathbf{9}$ & \multicolumn{2}{c}{$\mathbf{1 1 \times 1 1}$} \\
\hline Figure No. & $\begin{array}{c}2(\mathrm{a}), \\
8(\mathrm{a})\end{array}$ & $3(\mathrm{a})$ & $4(\mathrm{a})$ & $4(\mathrm{e})$ & $3(\mathrm{e})$ & $3(\mathrm{~m})$ & $4(\mathrm{i})$ & $3(\mathrm{i})$ & $4(\mathrm{~m})$ \\
\hline $\begin{array}{c}\text { Type of } \\
\text { Fabric sample }\end{array}$ & Twill & Plain & Twill & Plain & Twill & Plain & Twill & Twill & Twill \\
\hline $\begin{array}{c}\text { Yarn density } \\
\text { Warp } \times \text { Weft } \\
\text { Per inch }\end{array}$ & $\begin{array}{c}\text { Medium } \\
118 \times 60\end{array}$ & $\begin{array}{c}\text { High } \\
136 \times 72\end{array}$ & $\begin{array}{c}\text { Medium } \\
118 \times 60\end{array}$ & $\begin{array}{c}\text { High } \\
136 \times 72\end{array}$ & $\begin{array}{c}\text { Medium } \\
118 \times 60\end{array}$ & $\begin{array}{c}\text { Medium } \\
118 \times 60\end{array}$ & $\begin{array}{c}\text { Medium } \\
118 \times 60\end{array}$ & $\begin{array}{c}\text { Medium } \\
118 \times 60\end{array}$ & $\begin{array}{c}\text { Low } \\
110 \times 52\end{array}$ \\
\hline MR (\%) & 0 & 0 & 0 & 0 & 0.228 & 0.981 & 0 & 0 & 0 \\
\hline $\begin{array}{c}\text { Object } \\
\text { function }\end{array}$ & $\begin{array}{c}J_{1}\left(\mathbf{h}_{o p}\right), \\
J_{2}\left(\mathbf{h}_{o p}\right), \\
J_{3}\left(\mathbf{h}_{o p}\right) .\end{array}$ & $\begin{array}{c}J_{1}\left(\mathbf{h}_{o p}\right), \\
J_{2}\left(\mathbf{h}_{o p}\right) .\end{array}$ & $\begin{array}{c}J_{1}\left(\mathbf{h}_{o p}\right), \\
J_{2}\left(\mathbf{h}_{o p}\right) .\end{array}$ & $\begin{array}{c}J_{1}\left(\mathbf{h}_{o p}\right), \\
J_{2}\left(\mathbf{h}_{o p}\right) .\end{array}$ & $\begin{array}{c}J_{1}\left(\mathbf{h}_{o p}\right), \\
J_{2}\left(\mathbf{h}_{o p}\right) .\end{array}$ & $\begin{array}{c}J_{1}\left(\mathbf{h}_{o p}\right), \\
J_{2}\left(\mathbf{h}_{o p}\right) .\end{array}$ & $J_{3}\left(\mathbf{h}_{o p}\right)$. & $\begin{array}{c}J_{1}\left(\mathbf{h}_{o p}\right), \\
J_{2}\left(\mathbf{h}_{o p}\right) .\end{array}$ & $\begin{array}{c}J_{(}\left(\mathbf{h}_{o p}\right), \\
J_{2}\left(\mathbf{h}_{o p}\right), \\
J_{3}\left(\mathbf{h}_{o p}\right) .\end{array}$ \\
\hline
\end{tabular}

TABLE IV

EfFect on Defect Detection With the Variation in the Size of Smoothing Filter Size

\begin{tabular}{|c|c|c|c|c|c|c|c|c|c|c|c|c|c|c|c|c|}
\hline \multicolumn{3}{|c|}{ Size of Gaussian filter } & \multicolumn{2}{|c|}{$7 \times 7$} & \multicolumn{2}{|c|}{$9 \times 9$} & \multicolumn{2}{|c|}{$11 \times 11$} & \multicolumn{2}{|c|}{$13 \times 13$} & \multicolumn{2}{|c|}{$15 \times 15$} & \multicolumn{2}{|c|}{$17 \times 17$} & \multicolumn{2}{|c|}{$19 \times 19$} \\
\hline $\begin{array}{c}\text { Figure } \\
\text { No. }\end{array}$ & $\begin{array}{l}\text { Size of } \\
\text { optimal } \\
\text { filter }\end{array}$ & $\begin{array}{c}\text { Object } \\
\text { function }\end{array}$ & $\boldsymbol{P}_{\mathrm{d}}$ & $P_{\mathrm{n}}$ & $P_{\mathrm{d}}$ & $\boldsymbol{P}_{\mathrm{n}}$ & $\boldsymbol{P}_{\mathrm{d}}$ & $P_{\mathrm{n}}$ & $P_{\mathbf{d}}$ & $\boldsymbol{P}_{\mathbf{n}}$ & $P_{\mathbf{d}}$ & $\boldsymbol{P}_{\mathbf{n}}$ & $\boldsymbol{P}_{\mathbf{d}}$ & $\boldsymbol{P}_{\mathbf{n}}$ & $P_{\mathrm{d}}$ & $P_{\mathbf{n}}$ \\
\hline \multirow{3}{*}{2 (a) } & \multirow{3}{*}{$3 \times 3$} & $J_{1}\left(\mathbf{h}_{o p}\right)$ & 2093 & 0 & 2375 & 0 & 2420 & 0 & 2460 & 0 & 2474 & 0 & 2489 & 0 & 2494 & 0 \\
\hline & & $J_{2}\left(\mathbf{h}_{o p}\right)$ & 2093 & 0 & 2375 & 0 & 2420 & 0 & 2460 & 0 & 2474 & 0 & 2489 & 0 & 2494 & 0 \\
\hline & & $J_{3}\left(\mathbf{h}_{o p}\right)$ & 2093 & 0 & 2375 & 0 & 2420 & 0 & 2460 & 0 & 2474 & 0 & 2489 & 0 & 2494 & 0 \\
\hline \multirow{3}{*}{8 (a) } & \multirow{3}{*}{$3 \times 3$} & $J_{1}\left(\mathbf{h}_{o p}\right)$ & 3735 & 0 & 3912 & 0 & 3994 & 0 & 4019 & 0 & 4039 & 0 & 4052 & 0 & 4059 & 0 \\
\hline & & $J_{2}\left(\mathbf{h}_{o p}\right)$ & 3735 & 0 & 3912 & 0 & 3994 & 0 & 4019 & 0 & 4039 & 0 & 4052 & 0 & 4059 & 0 \\
\hline & & $J_{3}\left(\mathbf{h}_{o p}\right)$ & 1914 & 10 & 2330 & 0 & 2412 & 2 & 2438 & 0 & 2482 & 0 & 2506 & 0 & 2513 & 0 \\
\hline \multirow{3}{*}{3 (a) } & \multirow{3}{*}{$5 \times 5$} & $J_{1}\left(\mathbf{h}_{o p}\right)$ & 978 & 0 & 1155 & 0 & 1335 & 0 & 1450 & 0 & 1504 & 0 & 1524 & 0 & 1534 & 0 \\
\hline & & $J_{2}\left(\mathbf{h}_{O p}\right)$ & 978 & 0 & 1155 & 0 & 1335 & 0 & 1450 & 0 & 1504 & 0 & 1524 & 0 & 1534 & 0 \\
\hline & & $J_{3}\left(\mathbf{h}_{o p}\right)$ & 846 & 79 & 1255 & 109 & 1491 & 106 & 1595 & 99 & 1646 & 101 & 1658 & 99 & 1659 & 99 \\
\hline \multirow{3}{*}{4 (a) } & \multirow{3}{*}{$5 \times 5$} & $J_{1}\left(\mathbf{h}_{o p}\right)$ & 121 & 0 & 155 & 0 & 175 & 0 & 182 & 0 & 186 & 0 & 188 & 0 & 189 & 0 \\
\hline & & $J_{2}\left(\mathbf{h}_{o p}\right)$ & 121 & 0 & 155 & 0 & 175 & 0 & 182 & 0 & 186 & 0 & 188 & 0 & 189 & 0 \\
\hline & & $J_{3}\left(\mathbf{h}_{o p}\right)$ & 494 & 40 & 558 & 40 & 578 & 35 & 588 & 29 & 594 & 28 & 594 & 28 & 596 & 28 \\
\hline \multirow{3}{*}{$4(b)$} & \multirow{3}{*}{$5 \times 5$} & $J_{1}\left(\mathbf{h}_{o p}\right)$ & 54776 & 32 & 55233 & 25 & 55336 & 16 & 55406 & 17 & 55423 & 15 & 55436 & 14 & 55435 & 13 \\
\hline & & $J_{2}\left(\mathbf{h}_{o p}\right)$ & 54776 & 32 & 55233 & 25 & 55336 & 16 & 55406 & 17 & 55423 & 15 & 55436 & 14 & 55435 & 13 \\
\hline & & $J_{3}\left(\mathbf{h}_{o p}\right)$ & 47196 & 4393 & 53654 & 5700 & 55206 & 6190 & 55727 & 6375 & 55939 & 6484 & 56006 & 6.526 & 56025 & 6530 \\
\hline \multirow{3}{*}{$3(\mathrm{e})$} & \multirow{3}{*}{$7 \times 7$} & $J_{1}\left(\mathbf{h}_{o p}\right)$ & 1593 & 12 & 1664 & 9 & 1751 & 4 & 1821 & 2 & 1866 & 0 & 1871 & 0 & 1874 & 0 \\
\hline & & $J_{2}\left(\mathbf{h}_{o p}\right)$ & 1593 & 12 & 1664 & 9 & 1751 & 4 & 1821 & 2 & 1866 & 0 & 1872 & 0 & 1874 & 0 \\
\hline & & $J_{3}\left(\mathbf{h}_{O p}\right)$ & 157 & 6920 & 267 & 12267 & 362 & 16895 & 370 & 18163 & 390 & 18955 & 385 & 18966 & 385 & 19081 \\
\hline \multirow{3}{*}{$3(\mathrm{~m})$} & \multirow{3}{*}{$7 \times 7$} & $J_{1}\left(\mathbf{h}_{o p}\right)$ & 890 & 14 & 1020 & 15 & 1121 & 11 & 1174 & 11 & 1193 & 11 & 1191 & 11 & 1201 & 11 \\
\hline & & $J_{2}\left(\mathbf{h}_{o p}\right)$ & 890 & 14 & 1020 & 15 & 1121 & 11 & 1174 & 11 & 1193 & 11 & 1191 & 11 & 1201 & 11 \\
\hline & & $J_{3}\left(\mathbf{h}_{o p}\right)$ & 954 & 699 & 1384 & 1006 & 1769 & 1322 & 1957 & 14.31 & 2049 & 1500 & 2091 & 1524 & 2102 & 1532 \\
\hline \multirow{3}{*}{4 (i) } & \multirow{3}{*}{$9 \times 9$} & $J_{1}\left(\mathbf{h}_{o p}\right)$ & 226 & 1 & 272 & 0 & 311 & 0 & 330 & 0 & 337 & 0 & 339 & 0 & 341 & 0 \\
\hline & & $J_{2}\left(\mathbf{h}_{o p}\right)$ & 226 & 1 & 272 & 0 & 311 & 0 & 330 & 0 & 337 & 0 & 339 & 0 & 341 & 0 \\
\hline & & $J_{3}\left(\mathbf{h}_{o p}\right)$ & 374 & 1 & 526 & 0 & 610 & 0 & 655 & 0 & 679 & 0 & 690 & 0 & 690 & 0 \\
\hline \multirow{3}{*}{3 (i) } & & $J_{1}\left(\mathbf{h}_{o p}\right)$ & 286 & 3 & 343 & 0 & 365 & 0 & 390 & 0 & 405 & 0 & 414 & 0 & 414 & ) \\
\hline & $11 \times 11$ & $J_{2}\left(\mathbf{h}_{o p}\right)$ & 286 & 3 & 343 & 0 & 365 & 0 & 390 & 0 & 405 & 0 & 414 & 0 & 414 & 0 \\
\hline & & $J_{3}\left(\mathbf{h}_{o p}\right)$ & 762 & 13 & 1043 & 34 & 1102 & 33 & 1145 & 28 & 1161 & 27 & 1169 & 27 & 1171 & 27 \\
\hline & & $J_{1}\left(\mathbf{h}_{o p}\right)$ & 510 & 0 & 980 & 0 & 1125 & 0 & 1156 & 0 & 1171 & 0 & 1179 & 0 & 1187 & 0 \\
\hline $4(m)$ & $11 \times 11$ & $J_{2}\left(\mathbf{h}_{o p}\right)$ & 510 & 0 & 980 & 0 & 1125 & 0 & 1156 & 0 & 1171 & 0 & 1179 & 0 & 1187 & 0 \\
\hline & & $J_{3}\left(\mathbf{h}_{o p}\right)$ & 510 & 0 & 980 & 0 & 1125 & 0 & 1156 & 0 & 1171 & 0 & 1179 & 0 & 1187 & 0 \\
\hline
\end{tabular}




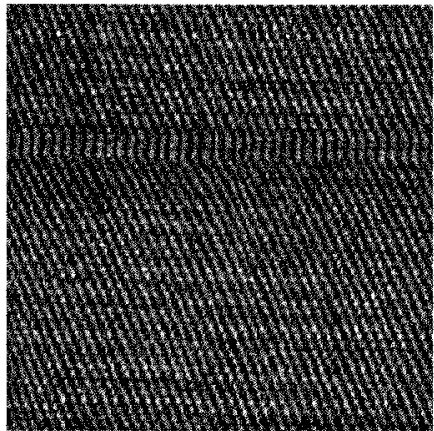

(a)

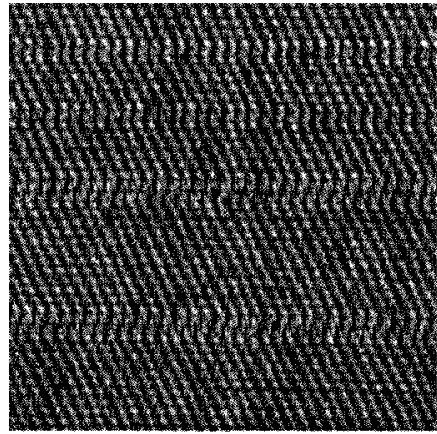

(d)

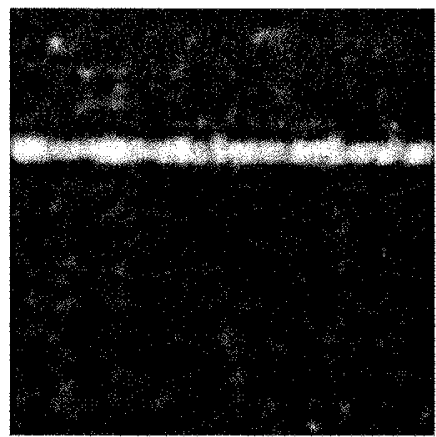

(b)

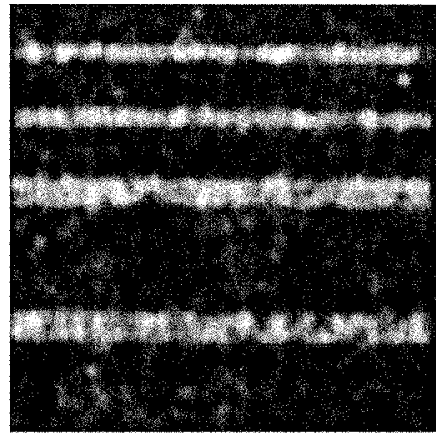

(e)

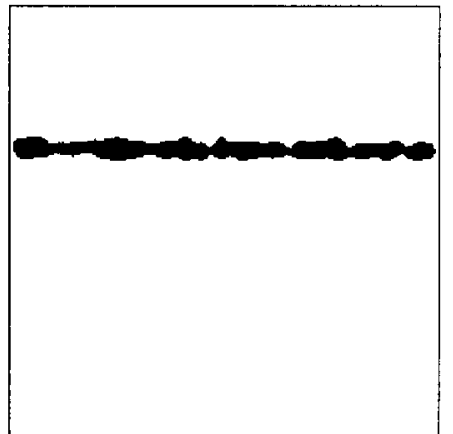

(c)

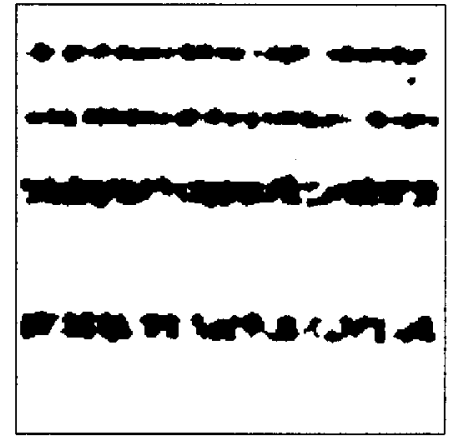

(f)

Fig. 5. Detection of mispick with $3 \times 3$ optimal filter mask. Fabric sample in (a) and (d); local energy estimate in (b) and (e); thresholded defects in (c) and (f).

The detection results for this defect can be improved (noise can be suppressed) with the optimal filter of size $11 \times 11$, instead of $9 \times 9$ is used in Fig. 4(n)-(o).

1) Effect of Smoothing Filter Size: High spatial resolution is required for the accurate preservation of edges; on the other hand high spatial frequency resolution is required for the accurate estimation of local energy. Since spatial and spatial frequency resolution is inversely related (uncertainty principle), accurate edge localization and accurate local energy estimation are conflicting goals [54]. The size of smoothing filter determines the tradeoff between the two conflicting goals. However, in material inspection, the detection of defects, i.e. estimation of local energy is more important than the localization of defects or the edges. Therefore, the goal of accurate defect localization or edge localization has not been considered in this work.

The size of smoothing filter used to obtain the minimum size masks in Table III was an ad-hoc choice. In order to observe the effect of variation of the smoothing filter size on performance, the experiments conducted in Section III-D were extended. The size of Gaussian smoothing filter was varied from $7 \times 7$ to $19 \times 19$ as shown in Table IV. The total number of pixels in the defect-free region $\left(P_{d}\right)$ and the region corresponding to the defect $\left(P_{n}\right)$ were observed for each of the smoothing filter sizes, while the size of optimal filter was fixed to minimum as computed in Table III. The thresholding limit for each of the defect was kept constant and was determined from the defect-free images. The results of the experiments, for all the three object functions, are summarized in Table IV.

With the increase in the size of smoothing filter from $7 \times 7$ to $19 \times 19$, the increase in pixels corresponding to defects $\left(P_{d}\right)$ can be observed for every defect in Table IV. However, this increase is large for some defects such as in Fig. 4(m) (132\%) while small for other defect such as negligible in Fig. 4(b) (1.2\%). The number of pixels in the defect-free region does not have any appreciable effect on the variation of the smoothing filter size, i.e. $P_{n}$ is almost constant. With the variation in the smoothing filter size, the minimum value of $M R<1 \%$ has been maintained for all the defects. The computational cost for increasing $P_{d}$, by increasing the size of smoothing filter is huge since the computational load for filtering (smoothing) is proportional to the square of mask size. Therefore, a compromise between the performance and the computational load (smoothing filter size), in the favor of later, is justified.

The detection results shown in Table IV for the defect in Fig. 4(b) have shown a small amount of noise, although the $M R$ is still less than 1. The optimal filters designed for some of the images, such as in Fig. 4(a), using the object function $J_{1}\left(\mathbf{h}_{o p}\right)$ or $J_{2}\left(\mathbf{h}_{o p}\right)$ results in zero noise, i.e. $P_{n}=0$. But the total number of pixels detected as defects $\left(P_{d}\right)$ from these optimal filters are much smaller (less than one third) than those for optimal filter designed by using the object function $J_{3}\left(\mathbf{h}_{o p}\right)$. Therefore, for such fabric defects, as in Figs. 4(a), 3(i), and 4(i), the optimal filters designed using the Fisher criterion $\left(J_{3}\left(\mathbf{h}_{o p}\right)\right)$ may be preferred due to the higher detection rate, i.e., $P_{d}$. Another observation can be observed from the Table IV for the detection results of defect in Fig. 3(e). The unusual detection results for the optimal filters designed with the Fisher criterion, i.e., $J_{3}\left(\mathbf{h}_{o p}\right)$, show that in all cases $P_{n} \gg P_{d}$. These results should be seen in conjunction with the image in Fig. 3(h), which suggests that this optimal filter emphasizes on defect-free region rather than on defect. Therefore the behavior of $P_{d}$ and $P_{n}$ has been interchanged. 


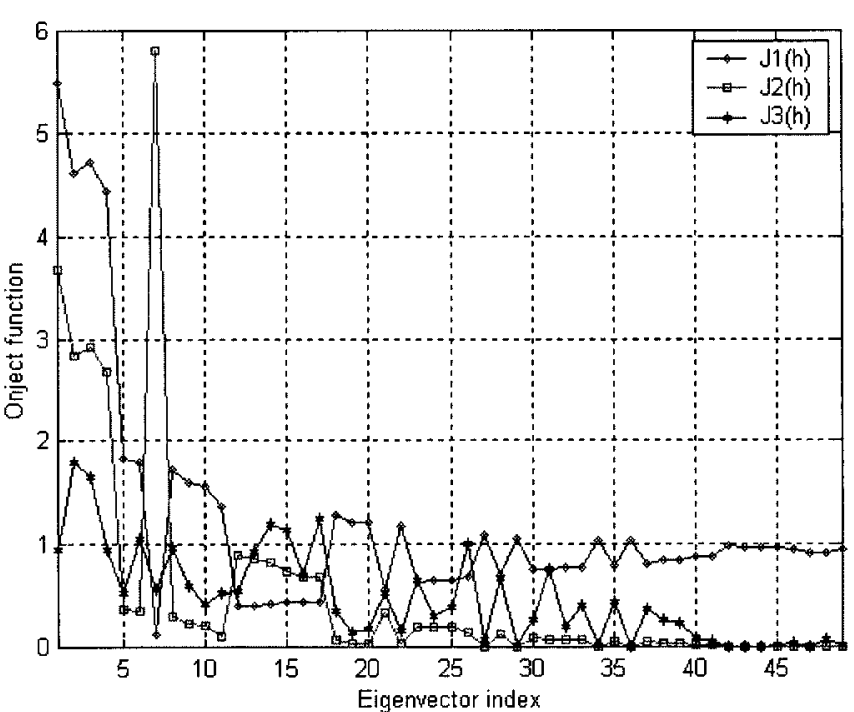

(a)

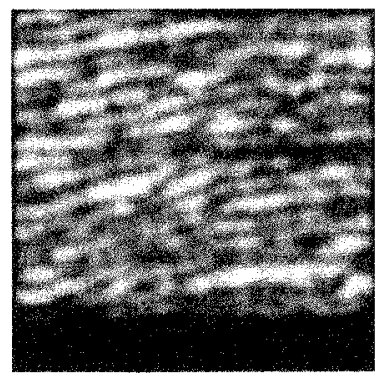

(b)

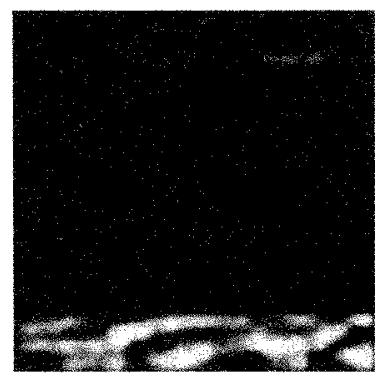

(c)
Fig. 6. (a) Object functions for the $7 \times 7$ optimal filters designed to detect the double-weft in Fig. 4(e). The corresponding local energy estimate from the optimal filter using designed by using $J_{1}\left(\mathbf{h}_{o} p\right)$ in (b) and by using $J_{2}\left(\mathbf{h}_{o} p\right)$ in (c).

\section{F. Discussion}

The results in Table IV suggests that the performance of optimal filters based on $J_{1}\left(\mathbf{h}_{o p}\right)$ and $J_{2}\left(\mathbf{h}_{o p}\right)$ for the detection of defects is same, i.e., $P_{d}$ and $P_{n}$ for these two object functions are exactly the same. However this may be only a co-incidence that the results from these two object functions for the minimum size mask were the same. The optimal filters (and the detection results) designed by using these two object functions, $J_{1}\left(\mathbf{h}_{o p}\right)$ and $J_{2}\left(\mathbf{h}_{o p}\right)$, can be entirely different as shown in Fig. 6.

Instead of $5 \times 5$ masks suggested in Table III, $7 \times 7$ optimal filters masks were designed to detect the defect double-weft shown in Fig. 4(e). Fig. 6(a) shows the object functions, $J_{1}\left(\mathbf{h}_{o p}\right)$ and $J_{2}\left(\mathbf{h}_{o p}\right)$, for each of the 49 eigenvectors analyzed to select the respective optimal filter. The first and the seventh eigenvector produces highest magnitude of the object function $J_{1}\left(\mathbf{h}_{o p}\right)$ and $J_{2}\left(\mathbf{h}_{o p}\right)$ respectively and is therefore the corresponding optimal filter. It can be noted that the magnitude of object function $J_{1}\left(\mathbf{h}_{o p}\right)$ for the seventh eigenvector is the smallest of all and is less than one. However, the same eigenvector produces the highest score for the object function
$J_{2}\left(\mathbf{h}_{o p}\right)$ among all the 49 eigenvectors. The local energy estimate for these two object functions is shown in Fig. 6(b)-(c). These results suggests that the optimal filter designed by using $J_{1}\left(\mathbf{h}_{o p}\right)$ emphasizes on the defect while that using $J_{2}\left(\mathbf{h}_{o p}\right)$ emphasizes on the defect-free region. Thus the results from the two object functions, $J_{1}\left(\mathbf{h}_{o p}\right)$ and $J_{2}\left(\mathbf{h}_{o p}\right)$ are different. The Fisher criterion in this experiment selected the second eigenvector as the optimal filter, which emphasizes on the defect.

The modeling and optimization of extracted features using any of the three criterion functions do not guarantee ${ }^{1}$ any specific feature response at defect boundaries. Therefore, depending on feature response at the boundaries of defects, the segmented defect may be severely biased with respect to their original position. Generally, this biasing was observed to be low and neglected for the reasons of computational simplicity. However, measures suggested by Randen et al. [54] can be used to avoid this biasing. Table IV suggests that the detection results with the Fisher criterion $J_{3}\left(\mathbf{h}_{o p}\right)$ have generated most of the noise i.e., $P_{n}$. One of the plausible reasons for poor performance of Fisher criterion function in some of the defects is the assumption used in deriving closed-form solution for the optimization [47].

Why does the performance change when the size of the optimal filter mask is varied? It is to be noted that the large (or small) eigenvectors (optimal filter) in (21) utilizes larger (or smaller) lags in the autocorrelation function. Thus the autocorrelation function of different size is required to compute the optimal filters of different sizes, which can have two different kinds of effect on the performance. In some cases, the small lags in the autocorrelation function may not be sufficient to model a texture or a defect. This may be a possible reason for the poor performance of the $3 \times 3$ or $5 \times 5$ masks in several cases. On the other hand, (too) larger lags in the autocorrelation function can be more susceptible to the noise due to the size of training data (image pitch) and/or due to the inhomogeneities. The optimal filters using such a large lag in the autocorrelation functions have not been designed to support any such conclusion. However, the results suggest that the size of optimal filter used to detect the defects have significant effect on the performance. The size of minimum mask suggested in Table III depends on the size and the nature (spectrum) of image pitch used to model the defect or the defect-free texture.

How subjective are the results? The optimal filters designed to detect the defects (in Table II) were found to be robust for the detection of same class of defects in other fabric sample (in Fig. 5). As long as the defect-free texture background does not change, the optimal filters designed to detect a class of defects are expected to perform well. However, the same class of defects may look differently at the different positions on the web, therefore some variation in the performance is intuitively expected.

\footnotetext{
${ }^{1}$ The texture in the images can be best approximated as wide sense stationary process within the texture, not at image boundaries.
} 


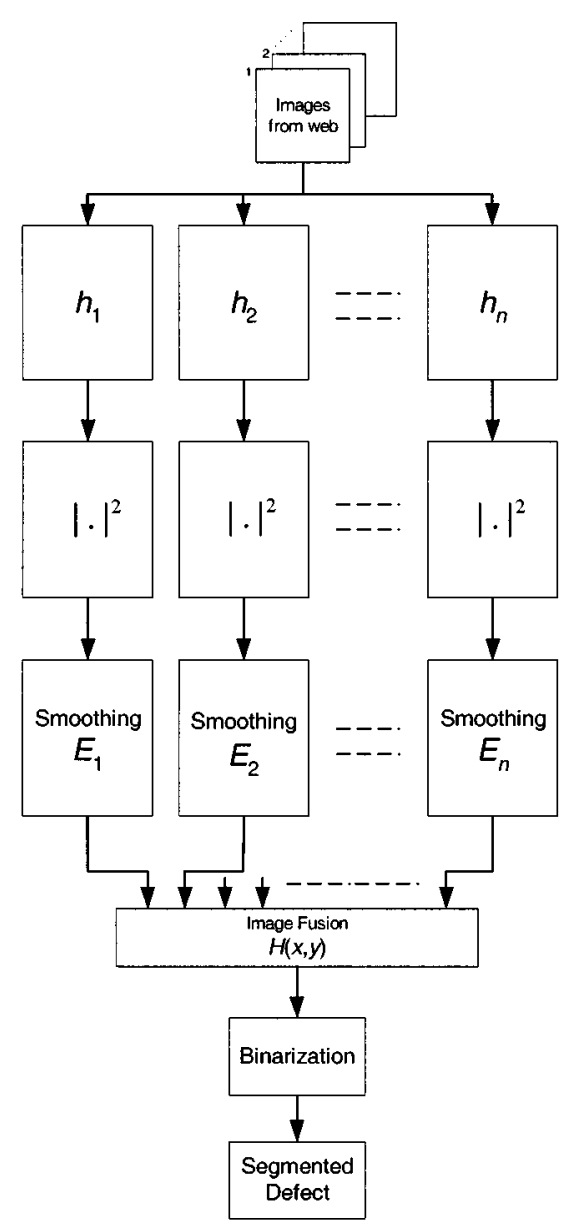

Fig. 7. Unsupervised web inspection using optimal filters.

\section{UNSUPERVISED DEFECT DETECTION}

Industrial web inspection in production lines requires continuous processing of images acquired from camera using backlighting. The orientation and the dimension of local defects generated in web materials, such as textile web, varies randomly and dynamically. Therefore, complete automation of visual inspection process requires unsupervised defect detection that can be used for the online web inspection. In this paper, the term 'unsupervised defect detection' refers to the detection of unknown class of defects for which there is no training. A general web inspection system using the optimal filters is shown in Fig. 7. The algorithm proposed in this section is divided into training and detection phases. The training process is offline and involves computations of optimal filters from the sample images with defects. The detection phase is an online process, in which the inspection images are inspected for the defect using precomputed optimal filters.

As shown in Fig. $7, h_{1} \ldots h_{n}$ is a set of $n$ minimal optimal filters required to capture the different class of defects for the required level of performance. The total number of filters depends on the range of defects to be detected (quality assurance) and the available computational complexity of the inspection system. For each of these optimal filtered outputs, the local energy estimate $\left(E_{1} \ldots E_{n}\right)$ is obtained using the process similar to as shown in Fig. 1.

\section{A. Image Fusion}

The function of the image fusion module is to integrate useful information from different channels, i.e., $E_{1} \ldots E_{n}$. Since each of the optimal filters $h_{1} \ldots h_{n}$ is designed to optimally suppress the defect-free texture with respect to a class of defect. The local energy estimates from each of these channels is unlikely to contain any component from the defect-free texture. Therefore a simple data fusion module involving vector addition of local energy estimates, rather than those used in [2] or [35], is adequate. Thus the fused image output $H(x, y)$, is obtained from the vector sum of local energy estimates from each of the channel, i.e.,

$$
H(x, y)=\sum_{j=1}^{n} E_{j}
$$

\section{B. Binarization}

The last stage is the thresholding of fused image output to generate a binary image of defects $B(x, y)$. A thresholding value is selected such that the value below this limit are considered as regular texture under inspection and value above are contributed from the defects. The simplest way to obtain this value is by the calibration at the beginning of inspection [3]. For calibration, a defect-free image (reference) sample is used to generate a fused image output $H_{r}(x, y)$. The threshold value $\phi_{t h}$ is obtained as follows:

$$
\phi_{t h}=\max _{x, y \in W}\left\{H_{r}(x, y)\right\}
$$

where " $W$ " is a window centered at the image $H_{r}(x, y)$. The window size is chosen to avoid the possible undesirable effects due to border distortions. In all experiments, the window size is obtained by removing 20 pixels (ad-hoc) from each side of the size of image $H_{r}(x, y)$.

\section{Experimental Setup and Results}

The online defect detection algorithm was evaluated using fabric samples gathered from the textile loom. The defects on the textile webs usually occur either in horizontal or in vertical direction [2]. This is due to the nature of weaving process in textile looms. Therefore, only two optimal filters, using $J_{1}\left(\mathbf{h}_{o p}\right)$ as the object function, corresponding to defect in the horizontal (weft) direction and vertical (warp) direction were designed. The $7 \times 7$ optimal filter mask $h_{1}$ was designed for fabric defect shown in Fig. 8(a) and its magnitude frequency response is shown in Fig. 8(f). Similarly, another fabric sample with the defect in vertical direction [Fig. 2(a)] was chosen and the $7 \times 7$ optimal filter $h_{2}$ was designed (results used from Section III-D) to segment the defect. The data fusion module using simple vector addition of local energy estimates $\left(E_{1} \ldots E_{n}\right)$ was used. The thresholding limit was obtained from a defect-free image as discussed in Section IV-B. 


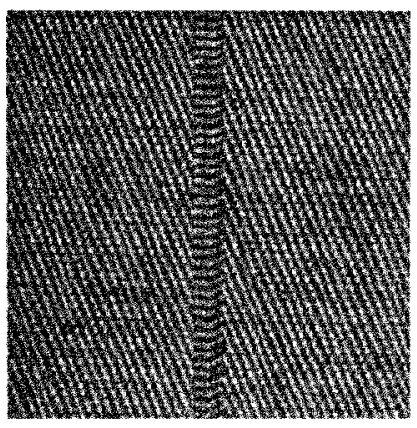

(a)

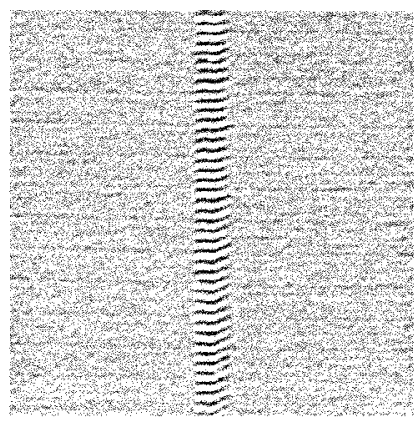

(b)

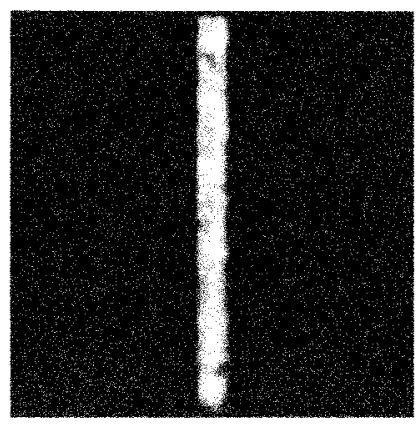

(c)

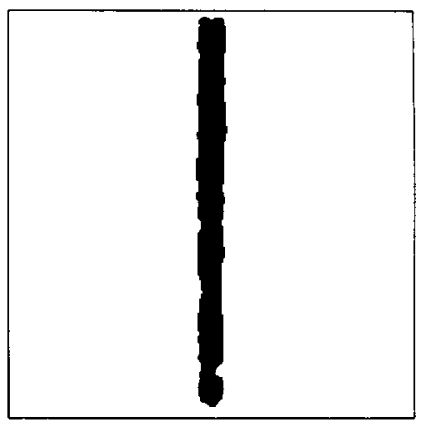

(d)

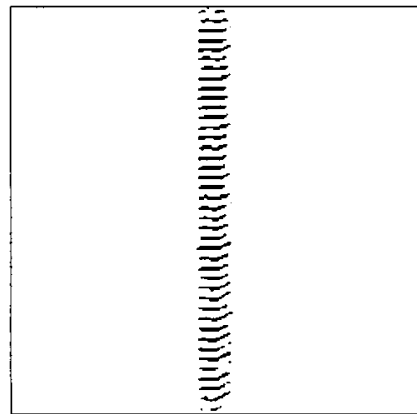

(e)

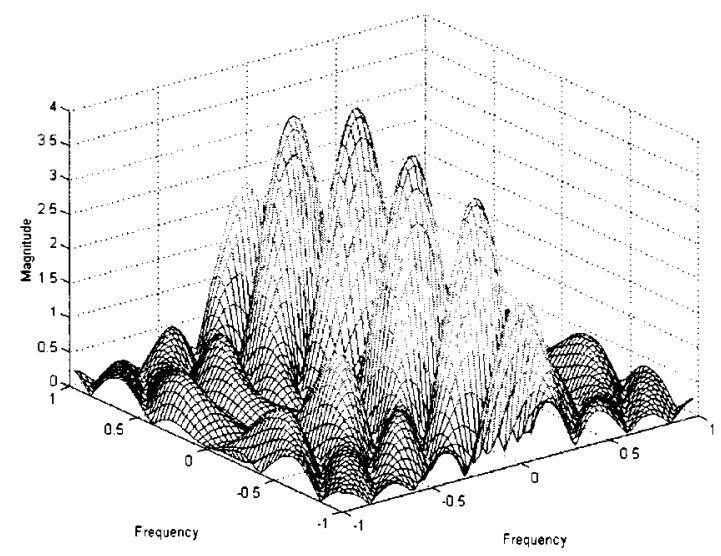

(f)

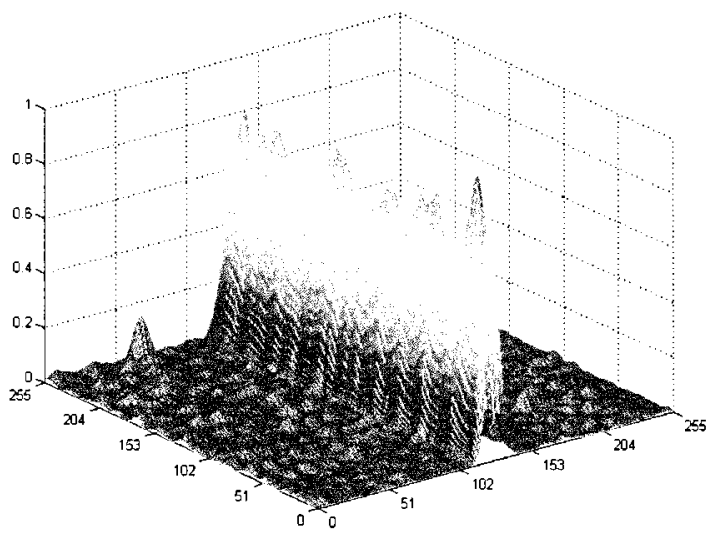

(g)

Fig. 8. (a) Fabric sample with a defect, (b) after filtering with optimal filter, (c) local energy estimate of image in (b), (d) segmented defect after thresholding image (c), (e) segmented defect after thresholding (b), (f) amplitude frequency response of $7 \times 7$ optimal filter, (g) 2 -D mesh plot of local energy estimate shown in $(\mathrm{c})$.

The fabric samples evaluated included most commonly occurring defects (e.g., mispick, double-weft, big-knot, netting multiples, slack-end, etc.). The fabric samples with these defects could be successfully detected and therefore the two-filter scheme proved to be robust. Some of the results with twill weave fabric samples are reproduced here.

Fig. 9(a) shows the defects (mispicks) in the vertical direction and therefore only filter $h_{2}$ contribute to the output 9 (c) and (d), as expected. For the defects shown in Fig. 10(a), con- tributions from $h_{1}$ and $h_{2}$ can be seen in Fig. 10(b) and (c) respectively. In Fig. 11(b) and (c), filter $h_{1}$ and successfully capture the components of defects in the two $h_{2}$ directions. Fig. 12 shows another example of robustness of the two filters in capturing defects in two directions. The components in this defect slack-end are evenly distributed in the horizontal and vertical directions. The respective components are captured in Fig. 12(b) and (c) and segmented defect can be seen in Fig. 12(e). 


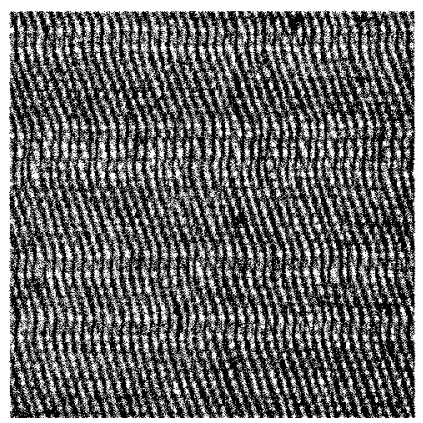

(a)

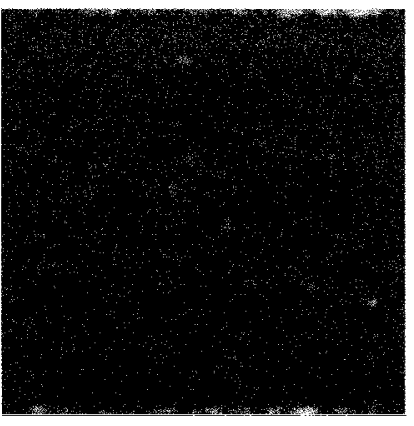

(b)

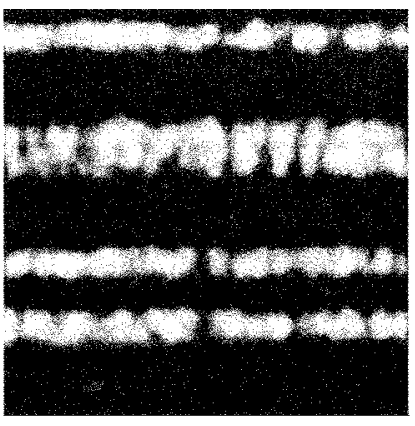

(c)

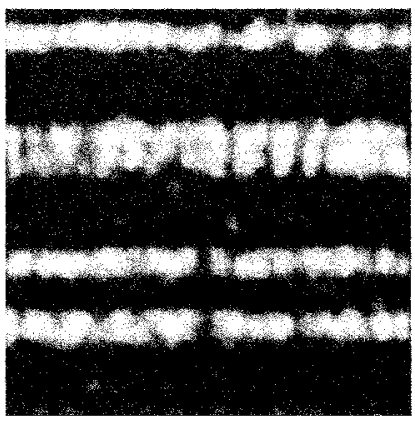

(d)

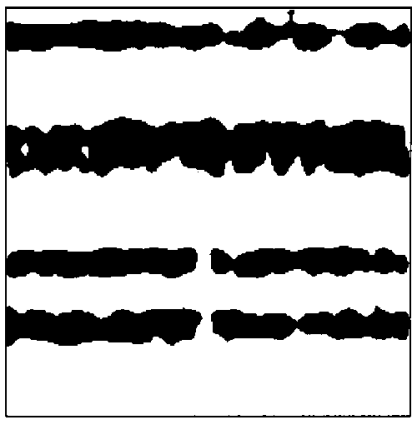

(e)

Fig. 9. (a) Fabric sample with defect, (b) output from filter $h_{1}$, (c) output from filter $h_{2}$, (d) combined output from $h_{1}$ and $h_{2}$, (e) segmented defect after thresholding image (d).

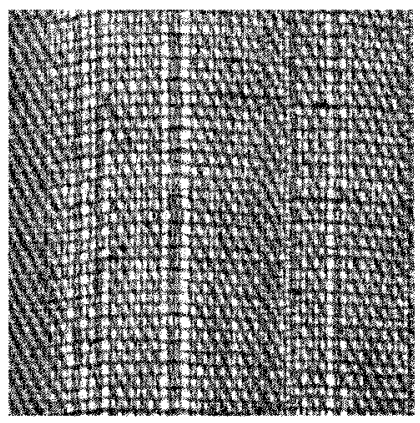

(a)

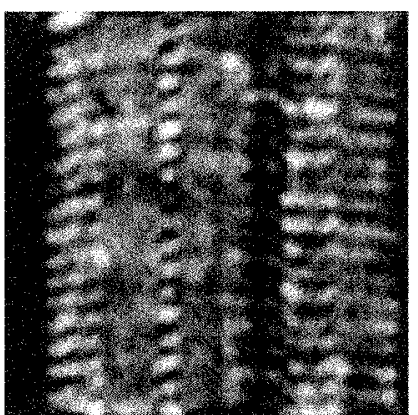

(b)

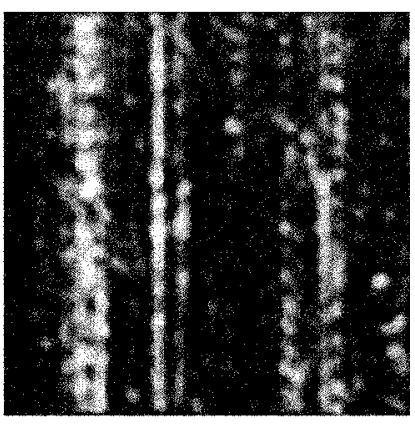

(c)

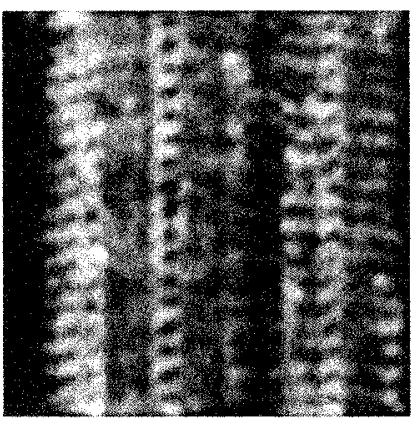

(d)

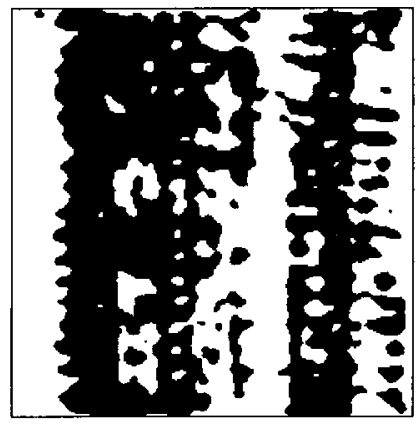

(e)

Fig. 10. (a) Fabric sample with defect, (b) output from filter $h_{1}$, (c) output from filter $h_{2}$, (d) combined output from $h_{1}$ and $h_{2}$, (e) segmented defect after thresholding image $(\mathrm{d})$. 


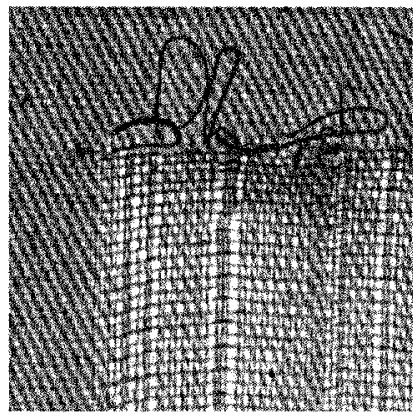

(a)

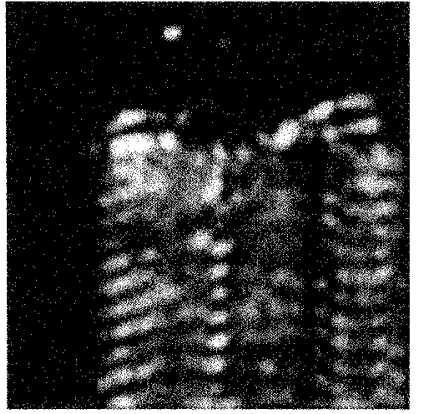

(b)

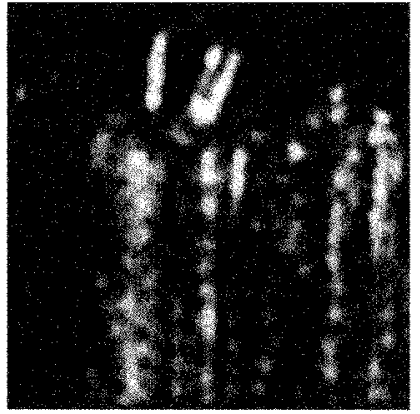

(c)

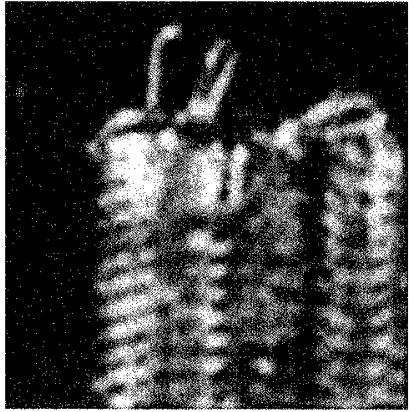

(d)

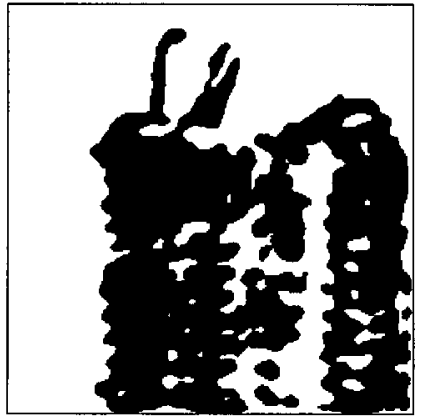

(e)

Fig. 11. (a) Fabric sample with defect, (b) output from filter $h_{1}$, (c) output from filter $h_{2}$, (d) combined output from $h_{1}$ and $h_{2}$, (e) segmented defect after thresholding image (d).

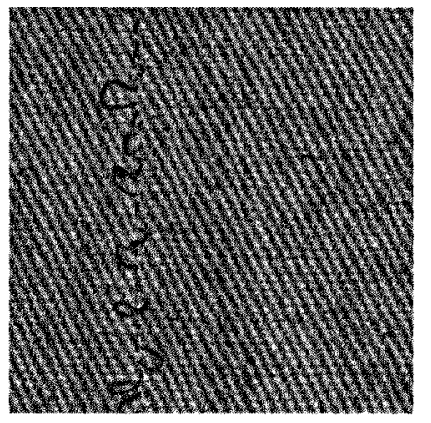

(a)

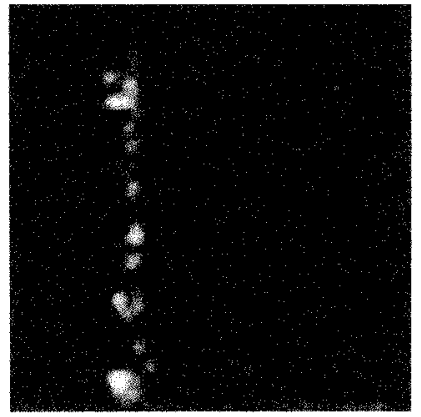

(b)

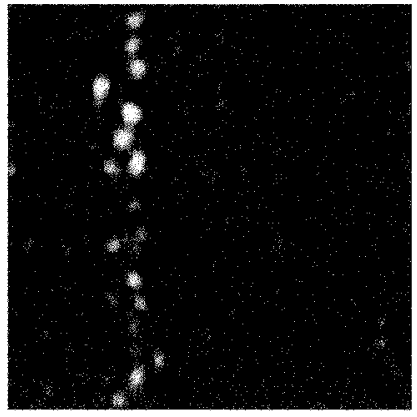

(c)

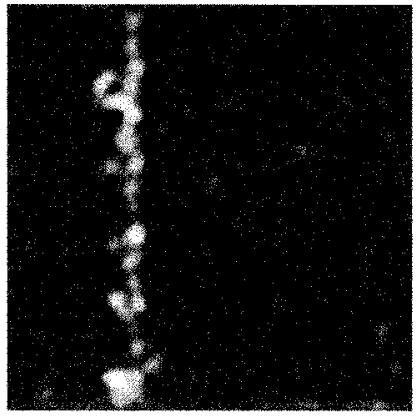

(d)

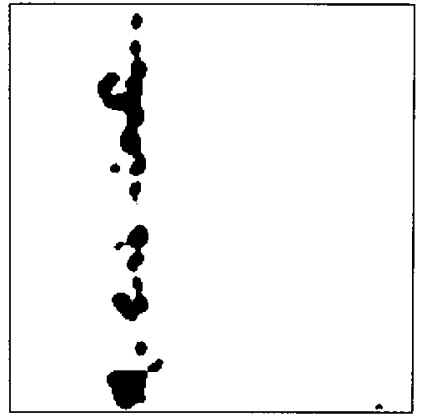

(e)

Fig. 12. (a) Fabric sample with defect, (b) output from filter $h_{1}$, (c) output from filter $h_{2}$, (d) combined output from $h_{1}$ and $h_{2}$, (e) segmented defect after thresholding image (d). 


\section{CONCLUSIONS}

In this paper, a new approach for the detection of defects using linear FIR filters with optimized energy separation has been investigated. The Mahalanobis-Singh [44], [45], Unser [46], and Fisher criterion [47], [48] functions were evaluated for the performance in fabric defect detection. The test conducted on different types of defect and different styles of fabric has yielded promising results.

One of the important conclusions of this work is that the size of optimal filter has appreciable effect on the performance for the defect detection. The texture segmentation work by Randen and Husøy [47], [48] has suggested that the optimal filters designed by with the Mahalanobis-Singh object function, i.e., $J_{1}\left(\mathbf{h}_{o p}\right)$, yields less robust results than those with the object functions $J_{2}\left(\mathbf{h}_{o p}\right)$ and $J_{3}\left(\mathbf{h}_{o p}\right)$. However the authors in [47], [48] have designed and used $7 \times 7$ optimal filters masks (with $32 \times 32$ Gaussian smoothing filter) and did not test their claim on the optimal filters of other sizes. Therefore, their conclusions are subjective to the size of the optimal filters they have used. Although the work in this paper is related to the defect detection, the robustness of the results with the object function $J_{2}\left(\mathbf{h}_{o p}\right)$ and $J_{3}\left(\mathbf{h}_{o p}\right)$ over the results with $J_{1}\left(\mathbf{h}_{o p}\right)$ (as claimed in [47]) could not be established. The results have shows that the optimal filters designed by using the object functions $J_{1}\left(\mathbf{h}_{o p}\right)$ and $J_{2}\left(\mathbf{h}_{o p}\right)$ give the same results, while in some cases the results with the object function $J_{3}\left(\mathbf{h}_{o p}\right)$ outweigh others. In order to keep this paper of manageable size, experimental results for different optimal filter mask sizes for different images have not been presented. The experimental results suggest that the performance with the object function $J_{1}\left(\mathbf{h}_{o p}\right)$ and $J_{2}\left(\mathbf{h}_{o p}\right)$ is the same in most cases. But this conclusion may be subjective and depend on the size of the optimal filter as shown in Fig. 6. Some excellent results for difficult defects i.e., defects with very subtle intensity variations, have been obtained with $J_{3}\left(\mathbf{h}_{o p}\right)$. Therefore, the Fisher criterion is recommended for the detection of such defects, but its performance too can be subjective to the size of the optimal filters as can be observed by comparing the results in Fig. 3(j)-(1) and Table III.

Another conclusion can be drawn from this work regarding the size of smoothing filter. The primary requirement in the web inspection problem is the detection of defects rather than their localization, i.e., size and location of the detected defects. The large size smoothing filters are computationally expensive and the performance does not increase much (Table IV) with the increase in the size of the smoothing filter. Prior work [47], [48] on texture segmentation employed $32 \times 32$ smoothing filter, which can be computationally expensive. The detection results (images) shown in this paper utilize $11 \times 11$ smoothing filter and can be a reasonable choice (Table III) for a given image resolution.

A general web inspection system using the optimal filters has been suggested in Section IV of this paper. The qualitative results in this paper have shown that the two-filter scheme is robust for a variety of fabric defects and yields promising results. The optimal and smoothing filters of different sizes can be used for the different channels in Fig. 7. Thus a user can obtain the desired performance $(M R)$ and/or the localization for the different defects. An averaging smoothing filter, instead of the Gaussian one used to show the experimental results in this paper, can be computationally economical for the online implementation of this system. The optimal filters can also be used to supplement the performance of the existing inspection systems that fail to detect a class of specific defects.

\section{REFERENCES}

[1] S. Kim, M. H. Lee, and K. B. Woo, "Wavelet analysis to defects detection in weaving processes," in Proc. IEEE Int. Symp. Industrial Electronics, vol. 3, July 1999, pp. 1406-1409.

[2] H. Sari-Sarraf and J. S. Goddard, "Vision systems for on-loom fabric inspection," IEEE Trans. Ind. Applicat., vol. 35, pp. 1252-1259, Nov./Dec. 1999.

[3] A. Kumar, "Automated defect detection in textured materials," $\mathrm{Ph} . \mathrm{D}$. thesis, Dept. Elect. Electr. Eng., The Univ. Hong Kong, Hong Kong, May 2001.

[4] A. Conci and C. B. Proença, "A fractal image analysis system for fabric inspection based on box-counting method," Comput. Networks ISDN Syst., vol. 30, pp. 1887-1895, 1998.

[5] L. Norton-Wayne, M. Bradshaw, and A. J. Jewell, "Machine vision inspection of web textile fabric," in Proc. Brit. Machine Vision Conf, Leeds, U.K., Sept. 1992, pp. 217-226.

[6] M. Bradshaw, "The application of machine vision to the automated inspection of knitted fabrics," Mechatronics, vol. 5, no. 2/3, pp. 233-243, 1995.

[7] L. Macaire and J. G. Postaire, "Flaw detection on galvanized metallic strips in real-time by adaptive thresholding," Proc. SPIE, vol. 2183, pp. 14-23, 1993.

[8] Y. F. Zhang and R. R. Bresee, "Fabric defect detection and classification using image analysis," Text. Res. J., vol. 65, pp. 1-9, Jan. 1995.

[9] T. Thomas and M. Cattoen, "Automatic inspection of simply patterned materials in the textile industry," Proc. SPIE, vol. 2183, pp. 2-12, Feb. 1994.

[10] D. Chetverikov, "Pattern regularity as a visual key," Image Vis. Comput., vol. 18, pp. 975-985, 2000.

[11] B. Mallick-Goswami and A. K. Datta, "Detecting defects in fabric with laser-based morphological image processing," Text. Res. J., vol. 70, pp. 758-762, Sept. 2000.

[12] W. J. Jasper and H. Potapalli, "Image analysis of mispicks in woven fabrics,” Text. Res. J., vol. 65, pp. 683-692, 1995.

[13] J. S. Lane, “Textile Fabric Inspection System,” U.S. Patent 5774 177, June 1998.

[14] A. Bodnarova, M. Bennamoun, and K. K. Kubik, "Defect detection in textile materials based on aspects of HVS," in Proc. IEEE SMC'98 Conf., San Diego, CA, Oct. 1998, pp. 4423-4428.

[15] M. Bennamoun and A. Bodnarova, "Automatic visual inspection and flaw detection in textile materials: Past, present and future," in Proc. IEEE Conf. on Systems, Man, and Cybernetics, 1998, pp. 4340-4343.

[16] L. H. Siew, R. M. Hodgson, and E. J. Wood, "Texture measures for carpet wear assessment," IEEE Trans. Pattern Anal. Machine Intell., vol. 10, pp. 92-105, Jan. 1988.

[17] R. W. Conners, C. W. McMillan, K. Lin, and R. E. Vasquez-Espinosa, "Identifying and locating surface defects in wood: Part of an automated lumber processing system," IEEE Trans. Pattern Anal. Machine Intell., vol. PAMI-5, pp. 573-583, Nov. 1983.

[18] I. Tsai, C. Lin, and J. Lin, "Applying an artificial neural network to pattern recognition in fabric defects," Text. Res. J., vol. 65, pp. 123-130, March 1995.

[19] A. Bodnarova, J. A. Williams, M. Bennamoun, and K. K. Kubik, "Optimal textural features for flaw detection in textile materials," in Proc. IEEE TENCON'97 Conf, Brisbane, Australia, Dec. 1997, pp. 307-310.

[20] M. Unser and F. Ade, "Feature extraction and decision procedure for automated inspection of textured materials," Pattern Recognit. Lett, vol. 2, pp. 181-191, March 1984.

[21] F. Ade, N. Lins, and M. Unser, "Comparison of various filter sets for defect detection in textiles," in Proc. 7th Int. Conf. Pattern Recognit, vol. 1, Montreal, QC, Canada, 1984, pp. 428-431.

[22] C. Neubauer, "Segmentation of defects in textile fabric," in Proc. 11th Int. Conf. Pattern Recognition, The Hague, The Netherlands, Aug. 1992, pp. 688-691. 
[23] F. G. B. De Natale, "Rank-order functions for the fast detection of texture faults," Intl. J. Pattern Recognit. Artif. Intell., vol. 10, no. 8, 1986.

[24] G. S. Desoli, S. Fioravanti, R. Fioravanti, and D. Corso, "A system for automated visual inspection of ceramic tiles," in Proc. Int. Conf. Industrial Electronics, Control and Instrumentation, IECON'93, vol. 3, 1993, pp. $1871-1876$.

[25] I. S. Tsai and M. C. Hu, "Automated inspection of fabric defects using an artificial neural networks," Text. Res. J., vol. 66, pp. 474-482, July 1996.

[26] D. M. Tsai and C.-Y. Heish, "Automated surface inspection for directional textures," Image Vis. Comput., vol. 18, pp. 49-62, 1999.

[27] C. H. Chan and G. Pang, "Fabric defect detection by Fourier analysis," IEEE Trans. Ind. Applicat., vol. 36, pp. 1267-1276, Sept./Oct. 2000

[28] D. C. Mead, H. L. Kasdan, and J. L. Dorrity, "Method for Automatic Fabric Inspection," U.S. Patent 4124 300, Nov. 1978.

[29] C. Castellini, F. Francini, G. Longobardi, and B. Tirbilli, "On-line quality control using optical Fourier transform," Opt. Lasers Eng., vol. 24, pp. 19-32, 1992

[30] C. Ciamberlini, F. Francini, G. Longobardi, P. Sansoni, and B. Tiribili, "Defect detection in textured materials by optical filtering with structured detectors and self-adaptable masks," Opt. Eng., vol. 35, pp. 835-844, Mar. 1996

[31] J. G. Campbell and F. Murtagh, "Automatic visual inspection of woven textiles using a two-stage defect detector," Opt. Eng., vol. 37, pp. $2536-2542$, Sept. 1988

[32] J. G. Campbell, A. A. Hasim, and F. D. Murtagh, "Flaw detection in woven textiles using space-dependent fourier transform," Univ. Ulster, Faculty of Informatics, U.K., Magee College Preprint INFM-97-004, 1997.

[33] J. Escofet, R. Navarro, M. S. Millan, and J. Pladelloreans, "Detection of local defects in textiles webs using Gabor filters," Opt. Eng., vol. 37, pp. 2297-2307, Aug. 1998.

[34] A. Kumar and G. Pang, "Defect detection in textured materials using Gabor filters," IEEE Trans. Ind. Applicat., vol. 38, pp. 425-440, Mar./Apr. 2002

[35] — - "Fabric defect segmentation using multichannel blob detectors," Opt. Eng., vol. 39, no. 12, pp. 3176-3190, Dec. 2000.

[36] J. G. Vachtsevanos, M. Mufti, and J. L. Dorrity, "Method and apparatus for analyzing an image to detect and identify defects," U.S. Patent 5815 198, Sept. 1998.

[37] W. J. Jasper, S. J. Garnier, and H. Potapalli, "Texture characterization and defect detection using adaptive wavelets," Opt. Eng., vol. 35, pp. 3140-3149, Nov. 1996.

[38] A. Kumar and G. Pang, "Identification of surface defects in textured materials using wavelet packets," in Proc. 36th IEEE/IAS Annu. Meeting, vol. 1, Chicago, IL, Sept. 2001, pp. 247-251.

[39] S. Özdemir and A. Erçil, "Markov random fields and karhunen-loève transforms for defect inspection of textile products," in Proc. IEEE Conf. Emerging Technologies and Factory Automation, EFTA'96, vol. 2, Nov. 1996, pp. 697-703.

[40] F. S. Cohen, Z. Fan, and S. Attali, "Automated inspection of textile fabrics using textural models," IEEE Trans. Pattern Anal. Machine Intell., no. 13, pp. 803-808, Aug. 1991.

[41] D. P. Brzaković, P. R. Bakić, N. S. Vujović, and Sari-Sarraf, "A generalized development environment for inspection of web materials," in Proc. IEEE Int. Conf. Robotics and Automation, Albuquerque, NM, Apr. 1997, pp. 1-8.

[42] D. P. Brzaković, P. R. Bakić, and A. Liakopoulos, "An approach to quality control of texture web materials," Proc. SPIE, vol. 2597, pp. 60-69, Oct. 1995.

[43] J. G. Campbell, C. Fraley, F. Murtagh, and A. E. Raftery, "Linear flaw detection in woven textiles using model-based clustering," Univ. Washington, Dept. Statistics, Seattle, WA, Tech. Rep., 1996.

[44] A. Mahalanobis and H. Singh, "Application of correlation filters for texture recognition," Appl. Opt., vol. 33, pp. 2173-2179, Apr. 1994.

[45] _ - "Correlation filters for texture recognition and applications to terrain-delimitation in wide-area surveillance," in Proc. Int. Conf. Acoustics, Speech, and Signal Processing., ICASSP'94, vol. 5, Apr. 1994, pp. $153-156$.

[46] M. Unser, "Local linear transforms for texture measurements," Signal Process., vol. 11, no. 1, pp. 61-79, 1986.
[47] T. Randen and J. H. Husøy, "Texture segmentation using filters with optimized energy separation," IEEE Trans. Image Processing, vol. 8, pp. 571-582, Apr. 1999.

[48] — " "Optimal texture filtering," in Proc. IEEE Int. Conf. Image Processing, vol. 1, Oct. 1995, pp. 374-377.

[49] K. Fukunaga, Statistical Pattern Recognition, 2nd ed. San Diego, CA: Academic, 1990

[50] A. K. Jain and F. Furrokhnia, "Unsupervised texture segmentation using Gabor filters," Pattern Recognit., vol. 23, pp. 1167-1186, Dec. 1991.

[51] M. Unser and M. Eden, "Nonlinear operators for improving texture segmentation based on features extracted by spatial filtering," IEEE Trans. Syst., Man, Cybern., vol. 20, pp. 804-815, July/Aug. 1990.

[52] A. Papoulis, Probability, Random Variables and Stochastic Processes, 3rd ed. New York: McGraw-Hill, 1991.

[53] T. Randen and J. H. Husøy, "Optimal texture filter design using feature extraction modeling," in Working papers from Høgskolen i Stavanger, no. 27, Stavanger, Norway, 1997.

[54] T. Randen, V. Alvestad, and J. H. Husøy, "Optimal filtering for unsupervised texture feature extraction," in Proc. SPIE Conf. Visual Communications and Image Process, Orlando, FL, Mar. 1996, pp. 430-440.

[55] K. Y. Song, M. Petrou, and J. Kittler, "Texture crack detection," Machine Vis. Applicat., vol. 8, no. 1, pp. 63-76, 1995.

Ajay Kumar (S'99-M'01) received the Ph.D. degree from The University of Hong Kong in May 2001.

He was with the Indian Institute of Technology (IIT), Kanpur, as Junior Research Fellow and at IIT, Delhi as Senior Scientific Officer before joining Indian Railways. He joined the Indian Railway Service of Signal Engineers (IRSSE) in 1993 and worked as Assistant Signal \& Telecom Engineer. He worked as Project Engineer at IIT (Kanpur) during 1996-1997 and as Assistant Professor at NIST, Berhampur, India, from September 1997 to September 1998. He was a Research Associate with The University of Hong Kong from December 1998 to August 1999. He completed his doctoral research at The University of Hong Kong in a record time of 21 months (September 1999 to May 2001). Currently, he is engaged in postdoctoral research in the Department of Computer Science, Hong Kong University of Science and Technology. His research interests include pattern recognition with the emphasis on biometrics and defect detection using wavelets, filter banks, general texture analysis and neural networks.

Dr. Kumar has been an executive council member of HKU IEEE student's branch (1999-2000).

Grantham K. H. Pang (S'84-M'86-SM'01) received the Ph.D. degree from the University of Cambridge, U.K., in 1986 for research in multivariable control system design and expert systems.

He was with the Department of Electrical and Computer Engineering, University of Waterloo, Waterloo, ON, Canada, from 1986 to 1996, and joined the Department of Electrical and Electronic Engineering, The University of Hong Kong in 1996. Since 1988, he published more than 120 technical papers and has authored or co-authored six books. His research interests include expert systems for control system design, intelligent control, intelligent transportation system, neural networks, control theory and computer-aided design. In 1994, he worked as a Senior Visiting Researcher at Hitachi Research Laboratory in Japan. He has acted as a consultant to many companies including Mitsubishi Electric Corp. in Japan, Northern Telecom and Imperial Oil Ltd. in Canada. He is an Editor of the International Journal of Intelligent Control and Systems and is an Editor of Control and Computers, published by the International Association of Science and Technology for Development (IASTED).

Dr. Pang was awarded the ICI Prize for authorship of the best paper on the application of the theory of control published in the Transactions of the Institute of Measurement and Control in 1989. He was the Organizing Chair of the 1996 IEEE Symposium on Computer-Aided Control System Design. He was appointed by the President of the IEEE Control Systems Society as the Chair of the Technical Committee on Computer-Aided Control System Design (1993-1995). He is a Chartered Electrical Engineer and a member of the IEE and HKIE. 